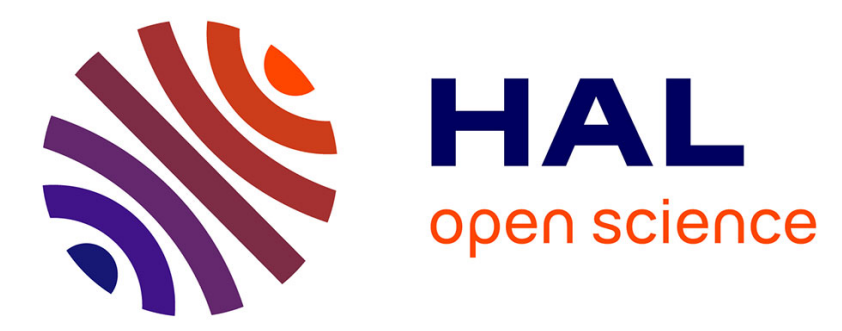

\title{
Numerical and experimental study of convective heat transfer in a vertical porous channel using a non-equilibrium model
}

W. Foudhil, B. Dhifaoui, S. Ben Jabrallah, A. Belghith, Jean Pierre Corriou

\section{- To cite this version:}

W. Foudhil, B. Dhifaoui, S. Ben Jabrallah, A. Belghith, Jean Pierre Corriou. Numerical and experimental study of convective heat transfer in a vertical porous channel using a non-equilibrium model. Journal of Porous Media, 2012, 15 (6), pp.531-547. 10.1615/JPorMedia.v15.i6.30 . hal-02383566

\section{HAL Id: hal-02383566 https://hal.science/hal-02383566}

Submitted on 27 Nov 2019

HAL is a multi-disciplinary open access archive for the deposit and dissemination of scientific research documents, whether they are published or not. The documents may come from teaching and research institutions in France or abroad, or from public or private research centers.
L'archive ouverte pluridisciplinaire HAL, est destinée au dépôt et à la diffusion de documents scientifiques de niveau recherche, publiés ou non, émanant des établissements d'enseignement et de recherche français ou étrangers, des laboratoires publics ou privés. 


\title{
Numerical and experimental study of convective heat transfer in a vertical porous channel using a
} non-equilibrium model

\author{
W. FOUDHIL ${ }^{a}$, B. DHIFAOUI ${ }^{a}$, S. BEN JABRALLAH ${ }^{a, b}$, A. BELGHITH ${ }^{a}$, J.P. $_{\text {CORRIOU }} *$ \\ ${ }^{a}$ Faculté des Sciences de Tunis, Laboratoire des Transferts de Chaleur et de Masse, \\ Campus Universitaire. 1060 Tunis, Tunisia \\ ${ }^{b}$ Faculté des Sciences de Bizerte. 7021. Bizerte, Tunisia. \\ ${ }^{c}$ Laboratoire Réactions et Génie des Procédés, CNRS-ENSIC-INPL, Nancy Université, \\ 1 rue Grandville, BP 20451, 54001 Nancy Cedex, France.
}

\begin{abstract}
Convective heat transfer in a vertical porous channel heated by the wall and isolated on the other face was simulated numerically and experimentally. The porous medium is formed by a solid matrix of spherical beads. The considered fluid is air that saturates the solid matrix. The two-temperature model and the Darcy-Brinkman-Forchheimer equation are adopted to represent this system and the porosity is considered as variable in the domain. The numerical model was used to analyze the effect of several operating parameters on heat transfer enhancement. Heat transfer decreases with the increase of the form factor. When Biot number increases, heat transfer between the heated wall and the porous domain is increased. Heat transfer increases with Reynolds

\footnotetext{
*Corresponding author. E-mail address: Jean-Pierre.Corriou@ensic.inpl-nancy.fr (J.P. Corriou)
} 
number and with the thermal conductivity of the solid matrix. The influence of the thermal conductivity of the particles on heat transfer in the porous medium decreases with increase of the thermal conductivity of the metallic beads, principally when the diameter of the beads increases. An increase of the bead diameter induces a decrease of heat transfer. Nusselt numbers based on the particle diameter have been correlated with respect to Reynolds number and the particle diameter. Furthermore, simulation results have been validated by experiments. Keywords : vertical channel, porous medium, heat transfer, convection

\section{Introduction}

Flows and heat transfer in porous media have been largely studied numerically and experimentally due to the importance of their applications. Heat transfer enhancement techniques play a very important role in thermal control technology used in microelectronic chips, thermal nuclear fusion, .... In this framework, several scientists have authored books about heat transfer phenomena in porous media, among whom Nield and Bejan (1999), Pop and Ingham (2001), Vafai (2005).

The choice of a flow law in a saturated porous medium such as Darcy, DarcyForchheimer or Darcy-Brinkman-Forchheimer models, is of prime importance for the formulation of the equations. Through literature analysis, Hlushkou and Tallarek (2006) present the different flow regimes in a porous medium, i.e. Darcy flow, inertial-viscous flow and turbulent flow, at both macroscopic and microscopic scales. The effects of the flows outside the domain of validity of Darcy law, corresponding to a flow velocity larger than $0.15 \mathrm{~m} / \mathrm{s}$, the variation of the porosity in the porous medium and the heat dispersion have been studied (Hsu and Cheng, 1990, Jiang et al., 1999a, 1996). The numerical studies of convection in porous media are based on two different models for the energetic balance, a model based on one energy equation where the local thermal 
equilibrium between the solid and liquid phases is established (one-temperature model), and a model based on two energy equations (two-temperature model) where a local non-equilibrium reigns between both phases (Mohamad, 2001, Slimi and BenNasrallah, 1997, Zhang et al., 2009).

Mohamad (2000) studied the validity of the equilibrium model for natural convection in an enclosure filled with a saturated porous medium. The results show that the equilibrium model is difficult to justify for non-Darcy regime and when the solid thermal conductivity is higher than the fluid thermal conductivity.

For the studies of forced convection in a porous channel, with a variable porosity, the two-temperature model is mostly used (Alazmi and Vafai, 2002, Amiri et al., 1995, Jiang and Lu, 2006, Jiang and Ren, 2001, Jiang et al., 1999a, 2004b) as it better describes the thermal state of the porous domain. However, there still remain open problems such as the boundary conditions used in this type of studies. In one of the first works, Amiri et al. (1995) considered two approaches. The first one assumes that the heat flux is divided between the phases, based on their effective conductivities and the temperature gradient

$$
\dot{q}_{w}=-\lambda_{f . e f f}\left(\frac{\partial T_{f}}{\partial y}\right)_{w}-\lambda_{s . e f f}\left(\frac{\partial T_{s}}{\partial y}\right)_{w} \quad \text { with: } \quad T_{f w}=T_{s w}=T_{w}
$$

The second one assumes that each phase receives the whole flux at the wall

$$
\dot{q}_{w}=-\lambda_{f . e f f}\left(\frac{\partial T_{f}}{\partial y}\right)_{w}=-\lambda_{s . e f f}\left(\frac{\partial T_{s}}{\partial y}\right)_{w}
$$

For numerical simulations of convective heat transfer in non-sintered porous media, Jiang and Ren (2001) show, by comparing the results of several approaches with experimental results, that the approach

$$
\dot{q}_{w}=-\lambda_{f}\left(\frac{\partial T_{f}}{\partial y}\right)_{w}=-\lambda_{s}\left(\frac{\partial T_{s}}{\partial y}\right)_{w}
$$

is the most adequate to describe the flux imposed at the wall for a local nonequilibrium model in the case of a horizontal channel filled by non-sintered porous media, heated by one wall and adiabatic at the other one. 
In the case of numerical simulation of convective heat transfer in sintered porous media, the boundary condition at the heated wall imposes that the fluid temperature is equal to that of the particle (Jiang and Lu, 2007). Several expressions can be found in literature, among which previous equation (1) and the following equations

in Martin et al. (1998)

$$
\dot{q}_{w}=-\left(\epsilon \lambda_{f}+(1-\epsilon) \lambda_{s}\right) \frac{\partial T}{\partial y} \quad \text { with: } \quad T_{f w}=T_{s w}=T_{w}
$$

in Lee and Vafai (1999)

$$
\dot{q}_{w}=-\epsilon_{w} \lambda_{f w}\left(\frac{\partial T_{f}}{\partial y}\right)_{w}-\left(1-\epsilon_{w}\right) \lambda_{s}\left(\frac{\partial T_{s}}{\partial y}\right)_{w} \quad \text { with: } \quad T_{f w}=T_{s w}=T_{w}
$$

in Jiang et al. (2004a)

$$
\dot{q}_{w}=-\lambda_{e f f} \frac{\partial T}{\partial y} \quad \text { with: } \quad T_{f w}=T_{s w}=T_{w}
$$

$\lambda_{\text {eff }}$ represents the effective conductivity of the porous domain (Zehner, 1970)

$$
\lambda_{e f f} / \lambda_{f}=[1-\sqrt{1-\epsilon}]+\frac{2 \sqrt{1-\epsilon}}{1-\sigma B}\left[\frac{(1-\sigma) B}{(1-\sigma B)^{2}} \ln \left(\frac{1}{\sigma B}\right)-\frac{B+1}{2}-\frac{B-1}{1-\sigma B}\right]
$$

such that $B=1.25((1-\epsilon) / \epsilon)^{10 / 9}$ and $\sigma=\frac{\lambda_{f}}{\lambda_{s}}$

By comparison with experimental results, Jiang et al. (2004a) showed that equation (6) yields satisfactory results, in the case of a study of convective heat transfer in sintered porous media.

Comparing eight approaches in the case of forced convection in a horizontal channel symmetrically heated, Alazmi and Vafai (2002) showed numerically that the physical properties of the fluid and of the solid have an impact on the choice of the employed model, and that both models converge in the case of a porosity $\epsilon=0.5$ and for a ratio of conductivities $\lambda_{f} / \lambda_{s}=1$.

The influence of the nature and the diameter of the particles forming the solid matrix on heat transfer has been studied by Wang and Du (1993) in the case of a vertical annular space, Nasr et al. (1994) in the case of a porous cylinder and Jiang and $\mathrm{Lu}$ (2006) in the case of a horizontal channel. Jiang et al. 
(1999b) performed an experimental study in the case of a horizontal channel in forced convection in the presence of water as coolant which shows that the heat transfer coefficient increases with the diameter of the glass particles and decreases with the diameter of metal particles. Pavel and Mohamad (2004) investigated experimentally and numerically the effect of metallic porous materials, inserted in a pipe, on heat transfer. They showed that higher heat transfer rates can be achieved using porous inserts at the expense of a reasonable pressure drop. Studies using air as the fluid crossing the porous medium are scarce. Hwang and Chao (1994) studied experimentally and numerically heat transfer of air in a porous medium and showed that the model with one-energy equation overestimates the Nusselt number. In the case of glass beads filling a horizontal channel, Jiang et al. (2004b) showed that heat transfer increases when the diameter of the particles decreases and when the thermal conductivity of the particles increases. The influence of the thermal conductivity of solid particles on the convective heat transfer of air in porous media decreases with increasing solid particle thermal conductivity.

Laguerre et al. (2006) performed an experimental study of heat transfer in a horizontal porous channel crossed by a flow at low velocity and showed that the air velocity and the position of the particles on the wall have a large influence on convective heat transfer between the heated wall of the cavity and air.

The physical and geometrical characteristics of a porous medium constituted by a packing of spherical beads are important parameters for the study of heat transfer in such media.

Pakdee and Rattanadecho (2009) studied transient natural convection flow through a fluid-saturated porous medium in a square enclosure with a partially cooling surface condition. The cavity, filled with porous medium, is insulated, except the top wall which is partially exposed to outside ambient temperature. It was found that the heat transfer coefficient, Rayleigh number, Darcy number, as well as flow direction strongly influenced flow characteristics and heat transfer 
mechanisms.

Vafai and Tien (1981) studied flow and heat transfer in a porous medium limited by a solid horizontal wall. They showed that the wall and inertia effects are more noticeable in highly permeable media, at large Prandtl numbers, for large pressure gradients and in the region close to the leading edge of the flow boundary layer. Lee et al. (2002) studied numerically the influence of the thickness of the dynamic limit layer on heat transfer in a channel filled with a porous medium. They obtained a correlation relating its thickness to Darcy and Reynolds numbers based on the permeability of the medium, showing that the decrease of the thickness increases the Nusselt number.

Experimental or numerical works concerning the transfer and flow in a vertical channel open at both extremities and filled with a porous medium are few. Among experimental works, Dhifaoui et al. (2007) studied energy storage in natural convection, Venugopal et al. (2010) also studied mixed convection intending to increase heat transfer in a porous channel filled with metallic beads. The numerical studies are recent and based on the model of local thermal equilibrium. In that way, Boutin and Gosselin (2009) studied heat transfer in a vertical porous channel limited by two vertical walls, one being exposed to solar radiation. Kumar et al. (2010) studied the stability of mixed convection in a vertical porous channel.

This literature review shows that most works performed in porous media deal with domains limited by horizontal walls and consider liquids as coolants. On the other side, the influences of the form factor and of the fluid-particles thermal exchanges on heat transfer are not considered in those studies.

The present work concerns convection in a vertical channel totally filled by a porous medium, crossed by air as the heat transfer fluid and heated on one of the walls by a constant heat flux density under local thermal non-equilibrium conditions. According to our knowledge, this has never been studied before.

The main objective of the present study is to analyze the effect of several 
operating parameters on heat transfer enhancement in porous media. The influences of the variation of Reynolds number, Biot number, the form factor, the diameter of the beads, and the thermal conductivity of the solid matrix on heat transfer, need to be further studied to optimize heat transfer enhancement using porous media. Correlations relating Nusselt number to Reynolds number and to the bead diameter are presented. The numerical study is validated by experiments.

\section{Nomenclature:}

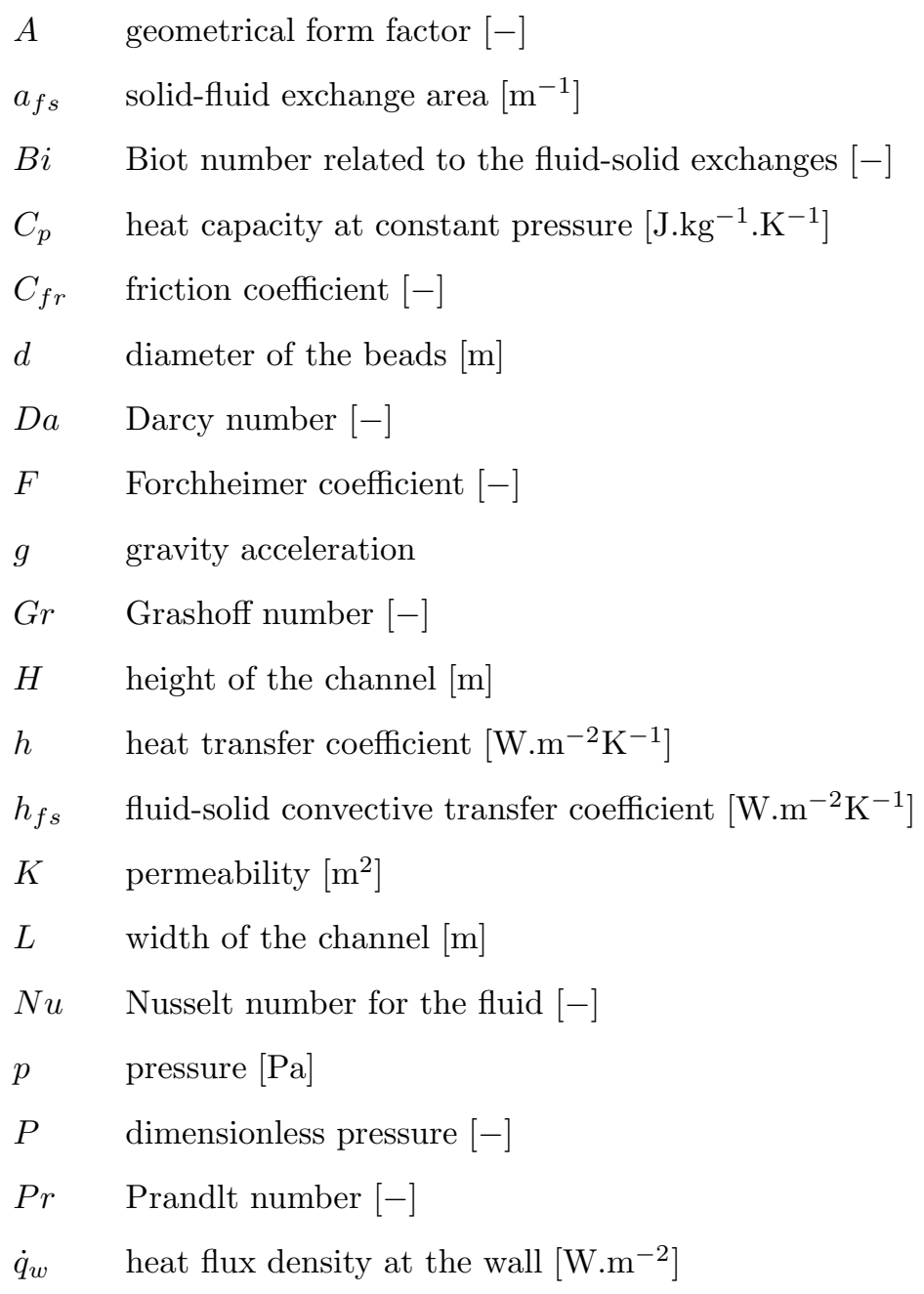


$R e_{p} \quad$ Reynolds number based on the diameter of the particle [-]

$R e_{L} \quad$ Reynolds number based on the width of the channel [-]

$R e_{K} \quad$ Reynolds number based on the permeability [-]

$r_{d L} \quad$ ratio between the diameter of the particles and the width of the channel [-]

$T$ temperature $[\mathrm{K}]$

$u, v \quad$ velocity components $\left[\mathrm{m} . \mathrm{s}^{-1}\right]$

$|\vec{w}| \quad$ norm of velocity $\left[\mathrm{m} . \mathrm{s}^{-1}\right]$

$U, V$ dimensionless velocity components $\left[\mathrm{m} . \mathrm{s}^{-1}\right]$

$|\vec{W}| \quad$ norm of dimensionless velocity [-]

$(x, y)$ cartesian coordinates $[\mathrm{m}]$

$(X, Y)$ dimensionless cartesian coordinates

\section{Greek symbols:}

$\beta \quad$ thermal expansion coefficient $\left[\mathrm{K}^{-1}\right]$

$\epsilon \quad$ porosity [-]

$\Lambda_{L} \quad$ inertia coefficient [-]

$\lambda_{f}$ thermal conductivity of the fluid $\left[\mathrm{W} \cdot \mathrm{m}^{-1} \cdot \mathrm{K}^{-1}\right]$

$\lambda_{s}$ thermal conductivity of the solid $\left[\mathrm{W} \cdot \mathrm{m}^{-1} \cdot \mathrm{K}^{-1}\right]$

$\lambda=\frac{\lambda_{s}}{\lambda_{f}}$ ratio of the heat conductivities [-]

$\mu \quad$ dynamic viscosity [Pa.s]

$\nu \quad$ kinematic viscosity $\left[\mathrm{m}^{2} . \mathrm{s}^{-1}\right]$

$\rho \quad$ density $\left[\mathrm{kg} \cdot \mathrm{m}^{-3}\right]$

$\theta \quad$ dimensionless temperature [-]

\section{Subscripts:}

$\begin{array}{ll}<> & \text { mean quantity } \\ e & \text { equivalent } \\ \text { eff } & \text { effective } \\ f & \text { fluid (air) } \\ m & \text { mean }\end{array}$




$\begin{array}{ll}0 & \text { reference } \\ p & \text { particle } \\ s & \text { solid } \\ t & \text { total } \\ w & \text { wall }\end{array}$

\section{Mathematical model}

The study concerns flow and heat transfer in a vertical porous medium constituted by two phases, a fluid phase of air and a porous solid not deformable phase of spherical beads.

A schematic representation of the physical system and its coordinates are represented in Fig. 1. The considered porous domain is a vertical channel open at both extremities, filled with homogeneous spherical beads, saturated by air as the fluid. The physical characteristics of both phases are summarized in Table 1. One of the walls of the channel is submitted to a constant heat flux density $\dot{q}_{w}$ and the other wall is thermally insulated. The main hypotheses adopted are that the porous medium is fixed, the flow is 2-dimensional, the fluid density is constant except when its variation directly causes Archimedes buoyancy forces (Boussinesq approximation) and radiative heat transfer is neglected.

Several authors consider that the dispersion term can be neglected in the case where the heat-conveying fluid in the porous medium is air (Calmidi and Mahajan, 2000). Consequently, in the present study, for simplification reasons, dispersion terms are not taken into account.

The stationary equations describing the flow and the transfers in the porous media at the macroscopic scale are based on the local volume-averaging technique proposed by Whitaker (1977). They include the continuity equation, the energy equations for the fluid and solid phases and the momentum equations 


\begin{tabular}{|c|c|c|c|c|}
\hline & $\begin{array}{c}\text { Thermal } \\
\text { conductivity } \\
\left(W \cdot m^{-1} \cdot K^{-1}\right)\end{array}$ & $\begin{array}{c}\text { Ratio of } \\
\text { conductivities } \\
\lambda=\lambda_{s} / \lambda_{f}\end{array}$ & $\begin{array}{c}\text { Beads } \\
\text { diameters } \\
(m)\end{array}$ & $\begin{array}{l}\text { Porosity of } \\
\text { porous } \\
\text { media }\end{array}$ \\
\hline Air & 0.025 & - & - & \multirow{5}{*}{$\begin{array}{c}\text { variable } \\
\text { (equation (13)) }\end{array}$} \\
\hline Glass & 0.7 & 28 & \multirow{4}{*}{$2.10^{-3}-10^{-2}$} & \\
\hline Stainless Steel & 16 & 640 & & \\
\hline Bronze & 75.35 & 300 & & \\
\hline Copper & 398 & 15307 & & \\
\hline
\end{tabular}

Table 1: Physical properties of the porous media

based on the Darcy-Brinkman-Forchheimer model with inertia and viscosity terms (Kaviany, 1999, Vafai and Sozen, 1990, Vafai and Tien, 1981)

Continuity equation

$$
\frac{\partial u}{\partial x}+\frac{\partial v}{\partial y}=0
$$

Energy balance for the fluid phase

$$
\left(\rho c_{p}\right)_{f}\left(u \frac{\partial T_{f}}{\partial x}+v \frac{\partial T_{f}}{\partial y}\right)=\epsilon \lambda_{f}\left(\frac{\partial^{2} T_{f}}{\partial x^{2}}+\frac{\partial^{2} T_{f}}{\partial y^{2}}\right)+h_{f s} a_{f s}\left(T_{s}-T_{f}\right)
$$

Energy balance for the solid phase

$$
0=(1-\epsilon) \lambda_{s}\left(\frac{\partial^{2} T_{s}}{\partial x^{2}}+\frac{\partial^{2} T_{s}}{\partial y^{2}}\right)+h_{f s} a_{f s}\left(T_{f}-T_{s}\right)
$$

Momentum equation on vertical axis

$$
\frac{\rho_{f}}{\epsilon}\left(\frac{\partial(u v / \epsilon)}{\partial x}+\frac{\partial(v v / \epsilon)}{\partial y}\right)=-\frac{\partial p}{\partial y}+\rho_{f} g-\left(\frac{\mu_{f}}{K}+\frac{\rho_{f} F \epsilon}{\sqrt{K}}|\vec{w}|\right) v+\frac{\mu_{f}}{\epsilon}\left(\frac{\partial^{2} v}{\partial x^{2}}+\frac{\partial^{2} v}{\partial y^{2}}\right)
$$

Momentum equation on horizontal axis

$$
\frac{\rho_{f}}{\epsilon}\left(\frac{\partial(u u / \epsilon)}{\partial x}+\frac{\partial(u v / \epsilon)}{\partial y}\right)=-\frac{\partial p}{\partial x}-\left(\frac{\mu_{f}}{K}+\frac{\rho_{f} F \epsilon}{\sqrt{K}}|\vec{w}|\right) u+\frac{\mu_{f}}{\epsilon}\left(\frac{\partial^{2} u}{\partial x^{2}}+\frac{\partial^{2} u}{\partial y^{2}}\right)
$$

The characteristic parameters (Alazmi and Vafai, 2002, Jiang and Ren, 2001, Kaviany, 1999) used in these equations are 
$K=\frac{d^{2} \epsilon^{3}}{150(1-\epsilon)^{2}}$, permeability of the porous medium

$F=\frac{1.75}{\sqrt{150} \epsilon^{1.5}}$, Forchheimer coefficient

$h_{f s}=\frac{\lambda_{f}}{d}\left(2+1.1 P r_{f}^{0.33}\left(\frac{v d}{\nu_{f}}\right)^{0.6}\right)$, fluid-solid convective heat transfer coefficient $a_{f s}=\frac{6(1-\epsilon)}{d}$, fluid-solid contact surface.

The porosity $\epsilon$ in the channel (Jiang and Ren, 2001) is

$$
\begin{aligned}
& \epsilon=\epsilon_{\infty}\left[1+1.7 \exp \left(\frac{-6 x}{d}\right)\right], \quad \text { if } \quad 0 \leq x \leq \frac{L}{2} \\
& \epsilon=\epsilon_{\infty}\left[1+1.7 \exp \left(\frac{-6(L-x)}{d}\right)\right], \quad \text { if } \quad \frac{L}{2} \leq x \leq L
\end{aligned}
$$

with $\epsilon_{\infty}=0.37 . L$ is the channel width.

The boundary conditions (Jiang and Ren, 2001, Jiang et al., 1999a, 1996) are the following

$$
\begin{aligned}
& \text { At } y=0: \quad u=0, \quad v=V_{0}, \quad T_{f}=T_{0}, \quad \lambda_{s, \text { eff }} \frac{\partial T_{s}}{\partial y}=h_{f s}\left(T_{s}-T_{0}\right) \\
& \text { At } y=H: \quad \frac{d u}{d y}=\frac{d v}{d y}=0, \quad \frac{\partial T_{f}}{\partial y}=\frac{\partial T_{s}}{\partial y}=0 \\
& \text { At } x=0: \quad u=v=0, \quad \dot{q}_{w}=-\lambda_{f}\left(\frac{\partial T_{f}}{\partial x}\right)_{w}=-\lambda_{s}\left(\frac{\partial T_{s}}{\partial x}\right)_{w} \\
& \text { At } x=L: \quad u=v=0, \quad \frac{\partial T_{f}}{\partial x}=\frac{\partial T_{s}}{\partial x}=0
\end{aligned}
$$

$T_{0}$ and $V_{0}$ are respectively the temperature and the velocity at the inlet of the porous domain.

All the variables in the previous equations (8-17), are transformed under dimensionless form by adopting the following definitions

$$
\begin{aligned}
& L_{r e f}=L ; P_{r e f}=\rho_{f} V_{0}^{2} ; V_{r e f}=V_{0} ; T_{r e f}=\frac{\dot{q}_{w} L}{\lambda_{f}} ; X=\frac{x}{L} ; Y=\frac{y}{L} ; A=\frac{H}{L} \\
& P=\frac{p-p_{0}}{\rho_{f} V_{0}^{2}} ; U=\frac{u}{V_{0}} ; V=\frac{v}{V_{0}} ; \theta=\frac{T-T_{0}}{\frac{\dot{q}_{w} L}{\lambda_{f}}}
\end{aligned}
$$


Under dimensionless form, the equations become

$$
\begin{aligned}
& \frac{\partial U}{\partial X}+\frac{\partial V}{\partial Y}=0 \\
& \frac{1}{\epsilon}\left(\frac{\partial(U V / \epsilon)}{\partial X}+\frac{\partial(V V / \epsilon)}{\partial Y}\right)=-\frac{\partial P}{\partial Y}-\left(\frac{r_{d L}}{R e_{p} D a}+\Lambda_{L}|\vec{W}|\right) V+ \\
& \frac{r_{d L}}{\epsilon R e_{p}}\left(\frac{\partial^{2} V}{\partial X^{2}}+\frac{\partial^{2} V}{\partial Y^{2}}\right)+\frac{r_{d L}^{2} G r_{f}}{R e_{p}^{2}} \theta_{f} \\
& \frac{1}{\epsilon}\left(\frac{\partial(U U / \epsilon)}{\partial X}+\frac{\partial(U V / \epsilon)}{\partial Y}\right)=-\frac{\partial P}{\partial X}-\left(\frac{r_{d L}}{R e_{p} D a}+\Lambda_{L}|\vec{W}|\right) U \\
& +\frac{r_{d L}}{\epsilon \operatorname{Re}_{p}}\left(\frac{\partial^{2} U}{\partial X^{2}}+\frac{\partial^{2} U}{\partial Y^{2}}\right) \\
& 0=\frac{r_{d L}(1-\epsilon)}{P r_{f} R e_{p}}\left(\frac{\partial}{\partial X}\left(\frac{\partial \theta_{s}}{\partial X}\right)+\frac{\partial}{\partial Y}\left(\frac{\partial \theta_{s}}{\partial Y}\right)\right)+\frac{r_{d L} B i}{\lambda P r_{f} R e_{p}}\left(\theta_{f}-\theta_{s}\right) \\
& U \frac{\partial \theta_{f}}{\partial X}+V \frac{\partial \theta_{f}}{\partial Y}=\frac{\epsilon r_{d L}}{P r_{f} R e_{p}}\left(\frac{\partial}{\partial X}\left(\frac{\partial \theta_{f}}{\partial X}\right)+\frac{\partial}{\partial Y}\left(\frac{\partial \theta_{f}}{\partial Y}\right)\right)-\frac{r_{d L} B i}{P r_{f} R e_{p}}\left(\theta_{f}-\theta_{s}\right)
\end{aligned}
$$

The boundary conditions are also transformed under dimensionless form yielding

$$
\begin{aligned}
& \text { At } Y=0: \quad U=0, \quad V=1, \quad \theta_{f}=0, \quad \frac{\partial \theta_{s}}{\partial Y}=\frac{B i}{\lambda(1-\epsilon) a_{f s} L} \theta_{s}, \\
& \text { At } Y=A: \quad \frac{d U}{d Y}=\frac{d V}{d Y}=0, \quad \frac{\partial \theta_{f}}{\partial Y}=\frac{\partial \theta_{s}}{\partial Y}=0, \\
& \text { At } X=0: \quad U=V=0, \quad\left(\frac{\partial \theta_{f}}{\partial X}\right)_{w}=-1, \quad \lambda\left(\frac{\partial \theta_{s}}{\partial X}\right)_{w}=-1, \\
& \text { At } X=1: \quad U=V=0, \quad \frac{\partial \theta_{f}}{\partial X}=\frac{\partial \theta_{s}}{\partial X}=0
\end{aligned}
$$

The dimensionless porosity is

$$
\begin{aligned}
& \epsilon=\epsilon_{\infty}\left[1+1.7 \exp \left(\frac{-6 X}{r_{d L}}\right)\right], \quad \text { if } \quad 0 \leq X \leq \frac{1}{2} \\
& \epsilon=\epsilon_{\infty}\left[1+1.7 \exp \left(\frac{-6(1-X)}{r_{d L}}\right)\right], \quad \text { if } \quad \frac{1}{2} \leq X \leq 1
\end{aligned}
$$

The dimensionless numbers defined in equations (18-27) are defined by

$$
\begin{aligned}
& G r_{f}=\frac{g \beta \dot{q}_{w} L^{4}}{\nu_{f}^{2} \lambda_{f}} ; \quad P r_{f}=\frac{\nu_{f}}{\alpha_{f}} ; \quad D a=\frac{K}{L^{2}} ; \quad \Lambda_{L}=\frac{F L}{\sqrt{K}} ; \\
& R e_{p}=\frac{V_{0} d}{\nu_{f}} ; \quad B i=\frac{h_{f s} a_{f s} L^{2}}{\lambda_{f}}
\end{aligned}
$$


and

$$
r_{d L}=d / L ; \quad \lambda=\lambda_{s} / \lambda_{f}
$$

where $G r_{f}, P r_{f}, D a, \Lambda_{L}, R e_{p}$ and $B i$ are respectively Grashoff number, Prandlt number, Darcy number, the inertia coefficient, Reynolds number, Biot number related to the fluid-solid exchanges. $r_{d L}$ and $\lambda$ are respectively the ratio between the diameter of the particles and the width of the channel and the ratio between the solid and fluid conductivities.

The Reynolds number based on the width of the channel can be used when the diameter of the particle is fixed or for the experimental results

$$
R e_{L}=\frac{V_{0} L}{\nu_{f}}
$$

Local Nusselt numbers related to the fluid and solid phases are given by the following expressions (Alazmi and Vafai, 2002, Amiri et al., 1995)

$$
N u_{f}=-\frac{L}{T_{f w}-T_{f m}}\left(\frac{\partial T_{f}}{\partial x}\right)_{x=0} \quad ; \quad N u_{s}=-\frac{L}{T_{s w}-T_{s m}}\left(\frac{\partial T_{s}}{\partial x}\right)_{x=0}
$$

where the mean temperatures are given by

$$
T_{f m}=\frac{1}{U_{m} L} \int_{0}^{L} v T_{f} d x \quad ; \quad T_{s m}=\frac{1}{L} \int_{0}^{L} T_{s} d x \quad \text { with: } \quad U_{m}=\frac{1}{L} \int_{0}^{L} v d x
$$

The mean values of Nusselt number are

$N u_{f m}=\frac{1}{H} \int_{0}^{H} N u_{f} d y \quad ; N u_{s m}=\frac{1}{H} \int_{0}^{H} N u_{s} d y \quad ; N u_{t m}=N u_{f m}+N u_{s m}$

Several authors (Demirel et al., 2000, Laguerre et al., 2006, Li and Finlayson, 1977) use a Nusselt number based on the diameter of particle, which would be expressed in the present case of the total mean Nusselt number as

$$
N u_{p}=N u_{t m} r_{d L}
$$




\section{Description of the experimental setup}

The experimental setup is mainly constituted by a vertical channel of planeparallel form and of form factor $A=H / L$. One of the vertical walls of this channel of dimensions $40 \times 20 \mathrm{~cm}$ is heated by a constant heat flux whereas the other wall is considered as adiabatic (Fig. 2). The channel is filled by a stack of glass beads to constitute the porous medium which is the object of the present study. The temperatures of the active walls forming the channel as well as the temperature in the porous medium are recorded by means of thermocouples of $K$ type of low diameter. The simultaneous distribution of the velocity and temperature of the heat-conveying fluid (air) crossing the solid matrix is measured by multifunctional sensors. This experimental device has already been tested in natural convection (Dhifaoui et al., 2007).

\section{Numerical solution}

The set of non linear partial differential equations is solved numerically by the finite volume method (Patankar and Spalding, 1972, Patankar, 1980). The grid is non uniform in both directions, expanding far from the boundaries, using a larger number of meshes in the neighborhood of the walls, at the inlet and outlet of the domain. The velocity-pressure coupling is solved by the algorithm SIMPLE (Patankar, 1980), and a power-law scheme is adopted to express the convective terms. The convergence criterion is satisfied when the temperature or velocity field difference between two successive iterations is lower than $10^{-6}$.

To examine the validity of the numerical scheme, our results have been compared with literature. In the following, for validation purposes, necessary adjustments have been introduced into our model, i.e. the channel is filled by the fluid (air), in the absence of a solid matrix, and heated at the walls by the same imposed temperature. The calculation code has been validated by comparison with the results of (Chang and Chang, 1996) in the case of a vertical channel 
in the absence of a solid matrix. Fig. 3 shows the variation of the velocity with respect to the width, at mid-height of the purely fluid channel. A good agreement between both lines is observed. The mean deviation and maximum absolute deviation are respectively equal to $1.110^{-4}$ and 0.0178 , this latter for a velocity equal to 0.57 .

To verify the independence of the simulation results with respect to the grid, the problem has been solved for several grid combinations presented in Table 2. It shows that the variation of the grid does not much influence the calculation results. The simulation is thus performed for a $95 \times 95$ grid.

\begin{tabular}{|c|c|c||c|}
\hline Grid & $N u_{f m}$ & $N u_{s m}$ & $N u_{t m}$ \\
\hline $75 \times 75$ & 73.37 & 4.42 & 77.80 \\
\hline $95 \times 95$ & 75.12 & 4.5 & 79.63 \\
\hline $125 \times 125$ & 76.94 & 4.57 & 81.51 \\
\hline
\end{tabular}

Table 2: Variation of the mean Nusselt numbers for the fluid, the solid and total, for different grids

The results of simulation have been compared to experiments for two different superficial velocities. Figures 4 and 5 present the comparison of simulation results from the model with those obtained by the experiments of the temperature of the fluid phase measured in the neighborhood of the heated wall, with respect to the height of the channel. The simulated and experimental lines present a deviation of $9 \%$ in the first half of the porous domain. This can be due to the presence of the metal grid which supports the solid matrix (Fig. 2) which somehow plays the role of a heat storage and thus leads to an increase of the medium temperature. In the second half of the domain, the deviation does not exceed $4 \%$. When comparing simulation results and experimental measurements, such a deviation is relatively common, being given the complexity of this kind of problem. 


\section{Results and discussion}

\section{$5.1 \quad$ Experimental results}

The pressure drop $\Delta P$ through a bed of spheres depends on the permeability, on the Forchheimer coefficient, on the seepage velocity of the fluid and on its flow properties.

Experimentally, $\Delta P$ is the pressure drop measured between two planes distant by $\Delta y$. In the range of velocities used in the present part, it was found experimentally that the pressure drop is nearly proportional to the length of the porous medium (Fig. 6). For a given height, the pressure drop increases with the superficial velocity.

The friction coefficient $C_{f r}$ of the fluid through the porous medium is calculated from the pressure drop $\Delta P$ in the porous channel and the mean velocity of the fluid $V_{0}$ (Wu and Hwang, 1998) as

$$
C_{f r}=\frac{\Delta P}{L} \frac{d}{\rho_{f} V_{0}^{2}} \frac{\epsilon^{3}}{(1-\epsilon)}
$$

The influence of Reynolds number $R e_{L}$ on the friction coefficient of the fluid through the porous medium is represented in Fig. 7. The friction coefficient decreases when Reynolds number (fluid velocity) increases especially at Reynolds numbers lower than around 10000, and becomes nearly constant at Reynolds numbers larger than 15000 .

\subsection{Numerical results and discussion}

\subsubsection{Influence of parameters on heat transfer}

Influence of Biot number In their experimental study concerning a horizontal channel with water as the fluid crossing the porous matrix, Jiang et al. (1999b) explained that convective heat transfer in the porous medium is controlled by two processes, convective heat transfer between the fluid and the 
heated wall and a "fine effect" of particles provoked by the spherical beads. This "fine effect" of the particles is controlled by the combined effects of the convective fluid-solid heat transfer and the conduction between the particles. This particle effect is enhanced in the case of beads of large thermal conductivity and large Reynolds numbers. In this study, Biot number characterizes heat exchanges between the fluid phase and the spherical particles. The influence of Biot number on the mean total Nusselt number is represented in two cases, the first one where the inlet velocity in the channel varies and the bead diameter is fixed $\left(r_{d L}=0.034\right.$, Fig. 8), the second one where the bead diameter varies and the inlet velocity in the channel is fixed $\left(R e_{L}=1500\right.$, Fig. 9). When heat exchange between air and the beads increases, causing an increase of Biot number, the total Nusselt number increases (linearly if the inlet velocity is varied and the bead diameter is fixed), i.e. heat transfer between the heated wall and the porous domain is increased.

The increase of Biot number is mainly caused by an increase of the inlet velocity or a decrease of the bead diameter, which induces an increase of heat transfer between the heated wall and the porous medium, as previously discussed. Consequently, an intensification of heat transfer between the fluid and the particles provokes an increase of transfer between the heated wall and the porous medium.

Influence of Reynolds number The influence of Reynolds number on the local Nusselt number relative to the exchanges between the heated wall and the fluid phase with respect to the channel height has been studied. The local heat transfer decreases along the heated wall in the flow direction whereas it increases with Reynolds number (Fig. 10). As a matter of fact, when the flow velocity increases, the thickness of the limit layer $\delta$ formed along the heated wall (Lee et al. (2002)) given by

$$
\frac{1}{\delta^{2}} \approx \frac{1+R e_{K}}{D a}
$$


decreases, and consequently the heat exchanges between the wall and the fluid increase, being given that

$$
R e_{K}=\frac{V_{0} K^{0.5}}{\nu_{f}}=\frac{R e_{p} D a^{0.5}}{r_{d L}}
$$

Lee et al. (2002) showed that at low Darcy numbers (like in the present case) the dimensionless thickness of the limit layer varies in a small domain $\delta \approx 10^{-3}$, however the chosen grid follows a variation which allows us to have several computational nodes in the limit layer.

The local Nusselt number varies rapidly close to the inlet of the channel whereas it remains nearly constant in the remaining domain, in agreement with Jiang et al. (1999b). In the neighborhood of the channel inlet, the temperature deviation between the fluid and the wall is relatively large, resulting in large values of Nusselt number. When the fluid progresses in the channel (Fig. 11), this temperature deviation is reduced and then the local Nusselt number takes low values.

Influence of the form factor The influence of Reynolds number on the mean total Nusselt number has been studied for different form factors (Fig. 12). The variation of the form factor is equivalent to varying the height of the porous domain while maintaining a uniform width between both walls. The mean Nusselt number increases when the form factor decreases. A relative increase of the channel height induces a heating of the medium as the exchange surface between the fluid and the heated wall increases (Fig. 13) and consequently the temperature difference between the heated wall and the porous medium decreases causing a decrease of heat transfer.

Influence of the thermal conductivity of the solid phase In this section, the nature of the solid matrix is examined. Different studied materials and their respective heat conductivities are presented in Table 1. 
The influence of nature of the solid matrix on the local Nusselt number of the fluid with respect to the height of the channel is studied for two different sizes of beads (Figs. 14 and 15). It shows that the choice of a high thermal conductivity material compared to glass enhances heat transfer. For solid matrices of important thermal conductivity, the thermal resistance of conduction between the particles is low compared to the fluid-solid convective resistance, thus the particle "fine effect" (Jiang et al., 1999b) is considerable.

On another side, the influence of the thermal conductivity of the particles on heat transfer in the porous medium decreases with the increase of the thermal conductivity of the metallic beads in agreement with Jiang et al. (2004b). This occurs as heat transfer of the heated wall towards the porous medium is ensured much more by the solid phase (large thermal conductivity) than by air. Consequently, the intensity of global heat transfer is mainly limited by the capacity of convective heat transfer of air. The comparison of Figs. 14 and 15 shows that this effect occurs principally when the diameter of the beads increases.

Influence of the bead diameter One of the parameters characterizing a porous medium is the diameter of the particles forming the solid matrix. Several physical expressions make use of this parameter such as the permeability of the medium, the fluid-solid convective transfer coefficient, the inertia coefficient.

The influence of the bead diameter on the local Nusselt number of the fluid with respect to the height of the channel (Fig. 16) shows that the local heat transfer between the heated wall and the fluid decreases along the heated wall according to the direction of the flow, and it increases when the particle diameter decreases. This result can be explained by the fact that the porous medium becomes more tortuous by decreasing the diameter of the beads, which favours heat transfer.

Heat transfer decreases when the diameter of the beads is increased (Fig. 17), all the more as the flow velocity is large. This result is valid for the different 
materials composing the particles (glass, stainless steel, bronze, copper).

In the case of glass beads and of water flow in the porous medium $\left(\lambda_{f} / \lambda_{s} \approx\right.$ 1), heat transfer increases with the diameter of the beads (Jiang et al., 1996, 1999b, Wang and Du, 1993). On the opposite, in the present case where the considered fluid is air $\left(\lambda_{f} \ll \lambda_{s}\right)$, heat transfer increases when the diameter of the beads decreases. For a channel filled with metallic beads (stainless steel, bronze, copper) crossed by air, heat transfer increases when the diameter of the beads decreases, in agreement with the results of Hwang and Chao (1994), Nasr et al. (1994) and Jiang et al. (1999a). As the contact surface between the particles and the fluid increases when the diameter of the particles decreases $\left(a_{f s}=6(1-\epsilon) / d\right)$, convective heat transfer is enhanced, leading to an increase of heat transfer between the heated wall and the porous medium. Indeed, it was previously shown in section 5.2.1 that an increase of the Biot number corresponding to the transfer between fluid and particles induced an increase of the mean total Nusselt number corresponding to heat transfer between the heated wall and the porous medium.

On another side, Jiang et al. (1999a) have shown that the variation of heat transfer with respect to the particle diameter can be related to the following criterion:

$$
\begin{cases}\text { If } & \rho_{0} v_{0} d>0.065 \lambda_{f}^{-4.82} \lambda_{e f f}^{5.82} / c_{p f}\left(\epsilon_{m} /\left(1-\epsilon_{m}\right)\right) \\ \text { then, } & \text { heat transfer increases with particle diameter } \\ \text { otherwise, } & \text { heat transfer increases when particle diameter decreases }\end{cases}
$$

$\lambda_{\text {eff }}$ represents the effective conductivity of the porous domain given by equation (7).

To check the validity of this criterion in the present study, the line comparing the left term with respect to the right term of criterion (35) (Fig. 18) is drawn for particles of different nature and diameter. Clearly, the second case of the criterion occurs for the different types of beads, thus heat transfer increases 
when the bead diameter decreases, which is verified in the present study. These results are in agreement with Jiang et al. (1999b) and Jiang et al. (2004b).

\subsubsection{Tables of results: summary}

In this section, tables gather the values of the mean total Nusselt number with respect to different operating parameters, such as Biot number, Reynolds number, the form factor, the conductivity of particles and their diameters.

The influence of Biot number on the mean total Nusselt number is presented in two cases, the first one where the inlet velocity in the channel varies and the bead diameter is fixed (Table 3a corresponding to Fig. 8), the second one where the bead diameter varies and the inlet velocity in the channel is fixed (Table 3b corresponding to Fig. 9). The mean total Nusselt number, thus the heat transfer increases with Biot number.

\begin{tabular}{|c|c|}
\hline$B i / 10^{4}$ & $N u_{t m}$ \\
\hline 4.07 & 89.33 \\
\hline 5.83 & 119.28 \\
\hline 7.26 & 143.85 \\
\hline 8.5 & 165.57 \\
\hline
\end{tabular}

(a) $r_{d L}=0.034$

\begin{tabular}{|c|c|}
\hline$B i / 10^{4}$ & $N u_{t m}$ \\
\hline 0.34 & 70.41 \\
\hline 0.49 & 73.08 \\
\hline 0.76 & 76.42 \\
\hline 1.42 & 81.05 \\
\hline
\end{tabular}

(b) $R e_{L}=1500$

Table 3: Variation of the mean total Nusselt number $N u_{t m}$ with respect to Biot number $B i\left(G r_{f}=10^{7}, P r_{f}=0.7, A=5, \lambda=28.57, D a=1.310^{-6}\right.$, $F=0.57$ ): (a) at constant bead diameter, (b) at constant inlet velocity

Table 4, corresponding to Fig. 12, presents the variation of the mean total Nusselt number with respect to Reynolds number for different form factors. The heat transfer increases with Reynolds number and decreases when the form factor increases. 


\begin{tabular}{|c||c|c|c|c|}
\hline & & & & \\
\hline
\end{tabular}

Table 4: Variation of the mean total Nusselt number $N u_{t m}$ with respect to Reynolds number $R e$ for different form factors $A\left(G r_{f}=10^{7}, P r_{f}=0.7\right.$, $\left.r_{d L}=0.067, \lambda=28.57, D a=5.210^{-6}, F=0.57\right)$

Table 5, corresponding to Fig. 17, presents the variation of the mean total Nusselt number with respect to the diameter of the beads for different ratios of fluid-solid conductivities. Heat transfer increases with the decrease of the diameter of the metallic or glass beads. Heat transfer increases with the thermal conductivity of the solid matrix.

\begin{tabular}{|c||c|c|c|c|c|c|c|c|}
\hline$r_{d L}$ & 0.033 & 0.05 & 0.067 & 0.083 & 0.1 & 0.116 & 0.13 & 0.167 \\
\hline \hline Glass & 119.28 & 95.17 & 81.05 & 71.39 & 64.28 & 58.78 & 54.37 & 47.71 \\
\hline Stainless steel & 196.51 & 146.23 & 117.71 & 99.03 & 85.81 & 75.94 & 68.30 & 57.30 \\
\hline Bronze & 232.10 & 163.4 & 127.44 & 105.1 & 89.84 & 78.75 & 70.32 & 58.37 \\
\hline Copper & 242.91 & 167.87 & 129.79 & 106.50 & 90.76 & 79.38 & 70.77 & 58.60 \\
\hline
\end{tabular}

Table 5: Variation of the mean total Nusselt number $N u_{t m}$ with respect to the diameter of the beads for different ratios of fluid-solid conductivities $\left(G r_{f}=10^{7}\right.$, $\left.\operatorname{Pr}_{f}=0.7, R e_{p}=100, A=5, F=0.57\right)$ 


\subsubsection{Proposition of correlations}

The heat transfer coefficient $N u_{p}$ between the wall and air increases with the air velocity (Fig. 19). Correlations of Nusselt number with respect to Reynolds number for $r_{d L}=0.034$ and for $\left(50 \leq R e_{p} \leq 200\right)$ are proposed

- for glass particles

$$
N u_{p}=0.52 R e_{p}^{0.44}
$$

- for metal particles

$$
N u_{p}=a R e_{p}^{b}
$$

with $a=2.28$ for stainless steel, 2.62 for bronze and 2.63 for copper. and $b=0.23$ for stainless steel, 0.24 for bronze and 0.25 for copper.

These correlations are compared in Fig. 20 to other correlations reported in the literature

$N u_{p}=1.56 \operatorname{Re}_{p}^{0.42} \operatorname{Pr}^{1 / 3}$ for metal spheres and air ; $100 \leq R e_{p} \leq 380$ and $d=3.8 \mathrm{~cm}$ (Laguerre et al. (2006)),

$N u_{p}=0.047 R e_{p}^{0.927}$ for polystyrene spheres and air ; $200 \leq R e_{p} \leq 1450$ and $0.13 \leq d / d_{e} \leq 0.22$ (Demirel et al. (2000)),

$N u_{p}=0.17 R e_{p}^{0.97}$ for metal spheres and air ; $20 \leq R e_{p} \leq 7600$ and $0.05 \leq$ $d / d_{e} \leq 0.3$ (Li and Finlayson (1977)).

The lines for the correlations shown in Fig. 20 are the result of a large range of conditions and show discrepancies between correlations. This can be explained by the difference of models (one- or two-temperature models) and conditions (particle diameter, range of Reynolds number, thermal conductivity of particle).

The comparison of the values of Nusselt numbers found in the present study for stainless steel with those of Li and Finlayson (1977) shows that they are close for $R e_{p} \approx 100$, but that the Reynolds exponent in Li and Finlayson (1977) correlation is much larger than the exponent in (37). This can be explained by the difference of the thermal model in use, the two-temperature model in 
the present case and one-temperature model for Li and Finlayson (1977), and possibly the thermal conductivity of unknown metal in Li and Finlayson (1977).

On another side, the difference between Nusselt values of the present study for glass with those of Demirel et al. (2000), for polystyrene for $R e_{p}=200$, can be explained by the fact that the thermal conductivity of glass is different from that of polystyrene. This is also the case concerning Nusselt number for metal as the present study compares stainless steel to the metal particles of Laguerre et al. (2006) who did not indicate the type of metal.

A non linear correlation equation for a glass particle with respect to $R e_{p}$ and $L / d$ is obtained as

$$
N u_{p}=2.5 R e_{p}^{0.41}(L / d)^{-0.41} \quad ; \quad 50 \leq R e_{p} \leq 200
$$

For air and polystyrene spheres, Demirel et al. (1999) obtained

$$
N u_{p}=0.0447 R e_{p}^{0.695}\left(d_{e} / d_{p}\right)^{-0.385} \quad ; \quad 200 \leq R e_{p} \leq 1450
$$

Equation (38) and equation (39) are represented for a given ratio $d / L=0.15$ (Fig. 21).

The values of Nusselt number obtained from equation (38) are larger than those obtained by equation (39). It can be explained by the low thermal conductivity of the polystyrene beads compared to the glass thermal conductivity.

\section{Conclusion}

A numerical study dealing with heat transfer in a vertical porous channel with a variable porosity has been conducted for a two-temperature model. This work shows that heat transfer increases with Reynolds number, decreases when the form factor increases, increases with the decrease of the diameter of the metallic or glass beads. Heat transfer is larger in the case of a matrix formed by metallic beads than with glass beads. This influence of the thermal conductivity of 
the particles on heat transfer of air in the porous medium decreases when the thermal conductivity of the particles increases, especially when the diameter of the beads is large. Nusselt numbers based on the particle diameter have been correlated with respect to Reynolds number and the particle diameter. A satisfying agreement between simulation and experimental results is obtained.

\section{References}

Alazmi, B. and Vafai, K., Constant wall heat flux boundary condition in porous media under local thermal non-equilibrium conditions, International Journal of Heat and Mass Transfer, vol. 45, pp. 3071-3087, 2002.

Amiri, A., Vafai, K. and Kuzay, T., Effects of boundary conditions on nonDarcian heat transfer through porous media and experimental comparison, Numerical Heat Transfer Part A, vol. 27, pp. 651-664, 1995.

Boutin, Y. and Gosselin, L., Optimal mixed convection for maximal energy recovery with vertical porous channel (solar wall), Renewable Energy, vol. 34, pp. 2714-2721, 2009.

Calmidi, V. V. and Mahajan, R. L., Forced convection in high porosity metal foams, Journal of Heat Transfer, vol. 122, pp. 557-567, 2000.

Chang, W. and Chang, W., Mixed convection in a vertical parallel-plate channel partially filled with porous media of high permeability, International Journal of Heat and Mass Transfer, vol. 39, pp. 1331-1342, 1996.

Demirel, Y., Abu-AlSaud, B., Al-Ali, H. and Makkawi, Y., Packing size and shape effect on forced convection in large rectangular packed ducts with asymmetric heating, International Journal of Heat and Mass Transfer, vol. 42, pp. 3267-3277, 1999. 
Demirel, Y., Sharma, R. and Al-Ali, H., On the effective heat transfer parameters in a packed bed, International Journal of Heat and Mass Transfer, vol. 43, pp. 327-332, 2000.

Dhifaoui, B., Jabrallah, S., Belghith, A. and Corriou, J., Experimental study of the dynamic behaviour of a porous medium submitted to a wall heat flux in view of thermal energy storage by sensible heat, International Journal of Thermal Sciences, vol. 46, pp. 1056-1063, 2007.

Hlushkou, D. and Tallarek, U., Transition from creeping via viscous-inertial to turbulent flow in fixed beds, Journal of Chromatography A, vol. 1126, pp. $70-85,2006$.

Hsu, C. and Cheng, P., Thermal dispersion in a porous medium, International Journal of Heat and Mass Transfer, vol. 33(8), pp. 1587-1597, 1990.

Hwang, G. and Chao, C., Heat transfer measurement and analysis for sintered porous channels, Journal of Heat Transfer, vol. 116, pp. 456-464, 1994.

Jiang, P., Li, M., Ma, Y. C. and Ren, Z. P., Boundary conditions and wall effect for forced convection heat transfer in sintered porous plate channels, International Journal of Heat and Mass Transfer, vol. 47 (10-11), pp. 20732083, 2004a.

Jiang, P. and Lu, X., Numerical simulation of fluid flow and convection heat transfer in sintered porous plate channels, International Journal of Heat and Mass Transfer, vol. 49, pp. 1685-1695, 2006.

Jiang, P. and Lu, X., Numerical simulation and theoretical analysis of thermal boundary characteristics of convection heat transfer in porous media, International Journal of Heat and Fluid Flow, vol. 28, pp. 1144-1156, 2007.

Jiang, P. and Ren, Z., Numerical investigation of forced convection heat transfer 
in porous media using a thermal non-equilibrium model, International Journal of Heat and Fluid Flow, vol. 22, pp. 102-110, 2001.

Jiang, P., Ren, Z. and Wang, B., Numerical simulation of forced convection heat transfer in porous plate channels using thermal equilibrium and nonthermal equilibrium models, Numerical Heat Transfer Part A, vol. 35, pp. 99-113, 1999a.

Jiang, P., Shi, G., Li, M. and Ren, Z., Experimental and numerical investigation of forced convection heat transfer of air in non-sintered porous media, Experimental Thermal and Fluid Science, vol. 28, pp. 545-555, 2004b.

Jiang, P., Wang, B., Luo, D. A. and Ren, Z., Fluid flow and convection heat transfer in a vertical porous annulus, Numerical Heat Transfer A 30, vol. 3, pp. 305-320, 1996.

Jiang, P., Wang, Z., Ren, Z. and Wang, B., Experimental research of fluid flow and convection heat transfer in plate channels filled with glass or metallic particles, Experimental Thermal and Fluid Science, vol. 20, pp. 45-54, 1999b.

Kaviany, M., Principles of Heat Transfer in Porous Media, Springer-Verlag, New York, 1999, second ed.

Kumar, J., Bera, P. and Khalili, A., Influence of inertia and drag terms on the stability of mixed convection in a vertical porous-medium channel, International Journal of Heat and Mass Transfer, vol. 53(23-24), pp. 5261-5273, 2010.

Laguerre, O., Amara, S. B. and Flick, D., Heat transfer betwen wall and packed bed crossed by low velocity airflow, Applied Thermal Engineering, vol. 26, pp. 1951-1960, 2006.

Lee, D., Jin, J. and Kang, B., Momentum boundary layer and its influence on 
the convective heat transfer in porous media, International Journal of Heat and Mass Transfer, vol. 45, pp. 229-233, 2002.

Lee, D. and Vafai, K., Analytical characterization and conceptual assessment of solid and fluid temperature differences in porous media, International Journal of Heat and Mass Transfer, vol. 42, pp. 423-435, 1999.

Li, C. and Finlayson, B., Heat transfer in packed beds-a reevaluation, Chemical Engineering Science, vol. 32, pp. 1055-1066, 1977.

Martin, A. R., Saltielb, C., Chaic, J. and Shyyd, W., Heat transfer enhancement with porous inserts in recirculating flows, Journal of Heat Transfer, vol. 120, pp. 458-467, 1998.

Mohamad, A., Non-Equilibrium, Natural Convection in a Differentially Heated Cavity Filled with a Saturated Porous Matrix, Journal of Heat Transfer, vol. 122 , pp. 380-384, 2000.

Mohamad, A., Natural Convection from a Vertical Plate in a Saturated Porous Media: Nonequilibrium Theory, Journal of Porous Media, vol. 4(2), pp. 181$186,2001$.

Nasr, K., Ramadhyani, S. and Viskanta, R., An experimental investigation on forced convection heat transfer from a cylinder embedded in a packed bed, Journal of Heat Transfer, vol. 116, pp. 73-80, 1994.

Nield, D. and Bejan, A., Convection in Porous Media, Springer, New York, 1999, second ed.

Pakdee, W. and Rattanadecho, P., Numerical Analysis of Natural Convection in Porous Cavities with Partial Convective Cooling Conditions., Journal of Porous Media, vol. 12(11), pp. 1083-1100, 2009. 
Patankar, S. and Spalding, D., A calculation procedure for heat, mass and momentum transfer in three dimensional parabolic flow, International Journal of Heat and Mass Transfer, vol. 15, pp. 1781-1806, 1972.

Patankar, S. V., Numerical heat transfer and fluids flow, Hemisphere, 1980.

Pavel, B. and Mohamad, A., An Experimental and Numerical Study on Heat Transfer Enhancement for gas Heat Exchangers Fitted with Porous Media, International Journal of Heat and Mass Transfer, vol. 47, pp. 4939-4952., 2004.

Pop, I. and Ingham, D., Convective Heat Transfer: Mathematical and Computational Modeling of Viscous Fluids and Porous Media, Pergamon, Oxford, 2001.

Slimi, K. and BenNasrallah, S., Transient natural convection in a vertical cylinder opened at the extremities and filled with a fluid saturated porous medium: validity of Darcy flow model and boundary layer approximations, International Journal of Heat and Mass Tansfer, vol. 41, pp. 1113-1125, 1997.

Vafai, K., ed., Handbook of Porous Media, Marcel Dekker, Boca Raton, Fl, 2005, second ed.

Vafai, K. and Sozen, M., Analysis of energy and momentum transport for fluid flow through a porous bed, Journal of Heat Transfer, vol. 112, pp. 690-699, 1990.

Vafai, K. and Tien, C., Boundary and Inertia Effect on flow and Heat Transfer in Porous Media, International Journal of Heat and Mass Transfer, vol. 24, pp. 195-203, 1981.

Venugopal, G., Balaji, C. and Venkateshan, S., Experimental study of mixed convection heat transfer in a vertical duct filled with metallic porous structures, International Journal of Thermal Sciences, vol. 49, pp. 340-348, 2010. 
Wang, B. and Du, J., Forced convective heat transfer in a vertical annuli filled with porous media, International Journal of Heat and Mass Transfer, vol. 36, pp. 4207-4214, 1993.

Whitaker, S., Simultaneous Heat, Mass and Momentum Transfer in Porous Media, a Theory of Drying, Advances in Heat Transfer, vol. 13, pp. 119-203, 1977.

Wu, C. and Hwang, G., Flow and heat transfer characteristics inside packed and fluidized beds, Journal of Heat Transfer, vol. 120(3), pp. 667-673, 1998.

Zehner, P., Wärmeleitfähigkeit von Schüttungen bei mässigen Temperaturen, Chemie Ingenieur Technik, vol. 42, pp. 933-941, 1970.

Zhang, X., Liu, W. and Liu, Z., Criterion for Local Thermal Equilibrium in Forced Convection Flow Through Porous Media, Journal of Porous Media, vol. 12(11), p. 2009, 2009. 


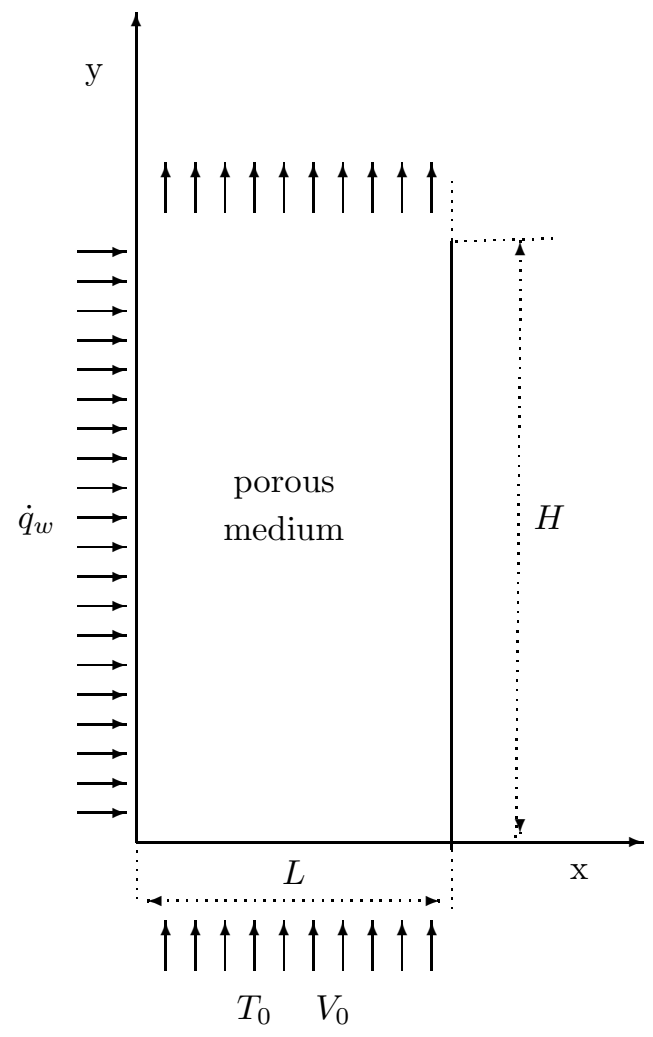

Figure 1: Schematic representation of the channel 


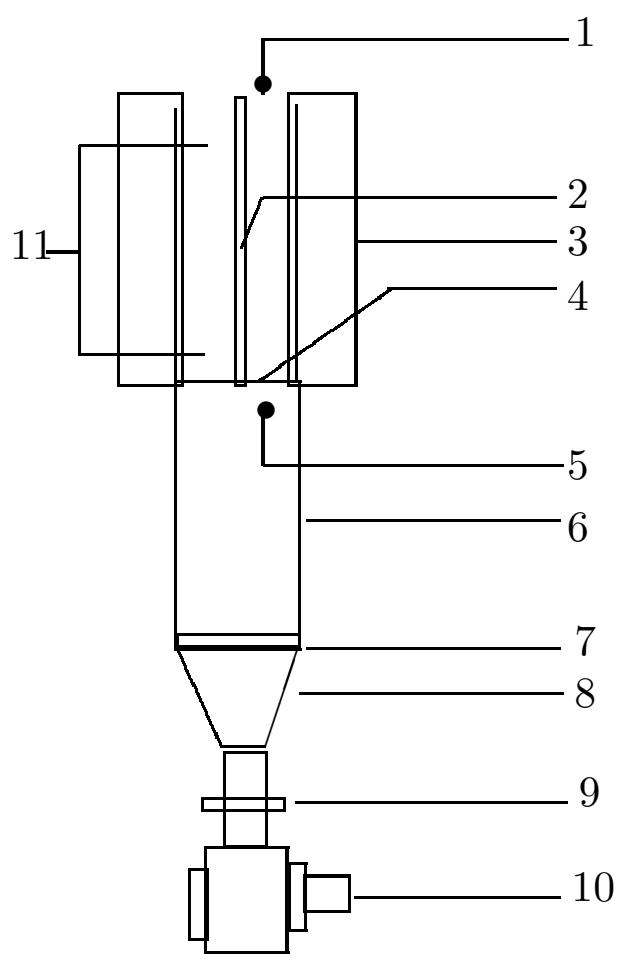

Figure 2: Simplified scheme of the experimental setup. 1: Temperature and velocity at the outlet, 2 : Heated wall, 3 : Insolating material (wood+cork), 4 : Grid (support of the solid matrix), 5: Temperature and velocity at the inlet, 6 : Pipe in plexiglas, 7 : Mesh for homogenization, 8 : Divergent, 9 : Diaphragm of variable section (flow rate variator), 10: Fan, 11: Differential pressure sensor. 


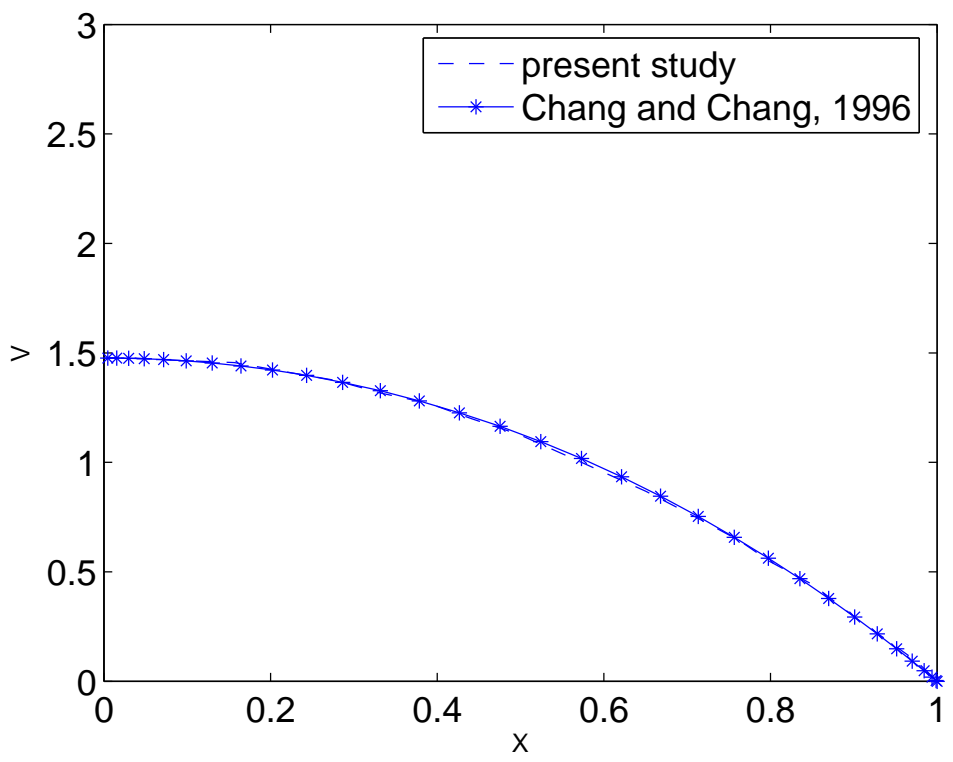

Figure 3: Comparison of the calculation code results with (Chang and Chang, 1996) for a vertical channel in the absence of a solid matrix: variation of the velocity with respect to the width, at mid-height of the domain. 


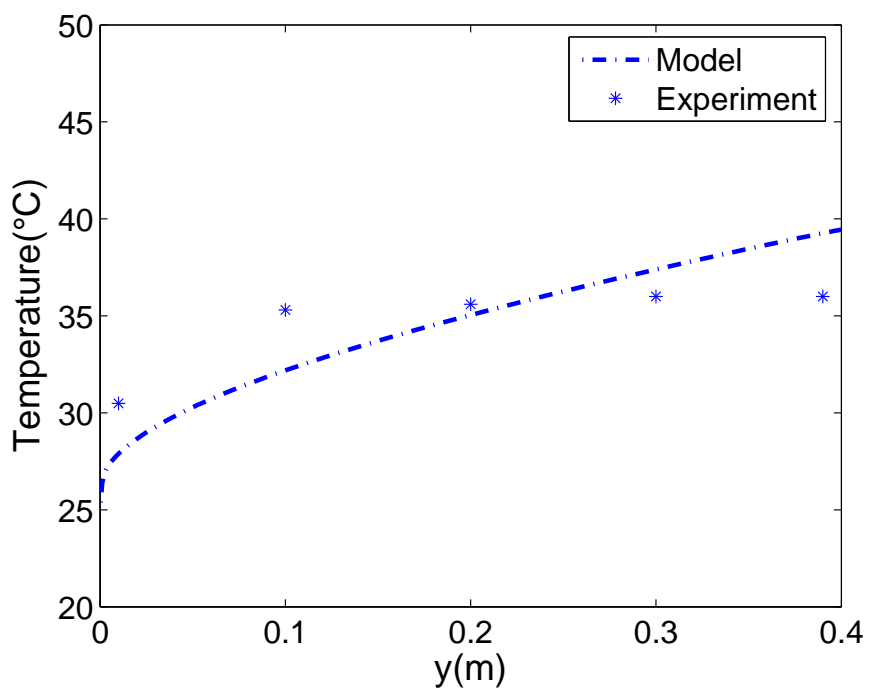

Figure 4: Comparison of the simulation results with the experiments: temperature profile in the neighborhood of the heated wall $\left(R e_{L}=3325, P r_{f}=0.7\right.$, $\left.G r_{f}=3.3510^{6}, A=10, r_{d L}=0.25, \lambda=28.57, D a=7.310^{-5}, F=0.57\right)$ 


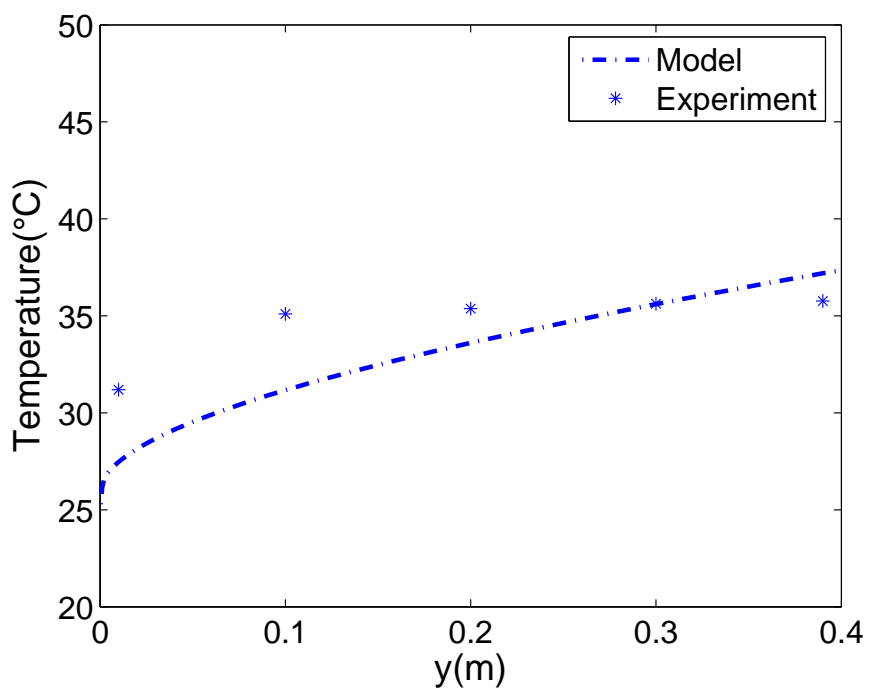

Figure 5: Comparison of the simulation results with the experiments: temperature profile in the neighborhood of the heated wall $\left(R e_{L}=4300, P r_{f}=0.7\right.$, $\left.\left.G r_{f}=3.3510^{6}, A=10, r_{d L}=0.25, \lambda=28.57, D a=7.310^{-5}, F=0.57\right)\right)$ 


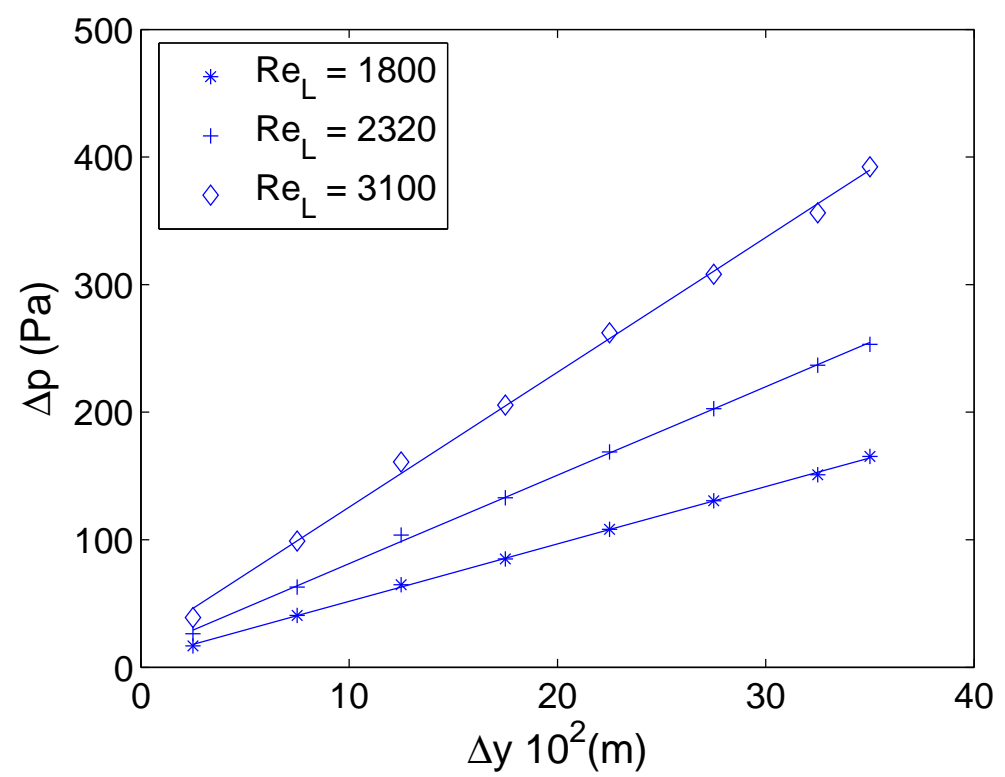

Figure 6: Variation of the pressure drop in the medium in the flow direction for different values of the velocity $\left(r_{d L}=0.25, P r_{f}=0.7, A=10, G r_{f}=3.3510^{6}\right.$, $\left.D a=7.310^{-5}\right)$ 


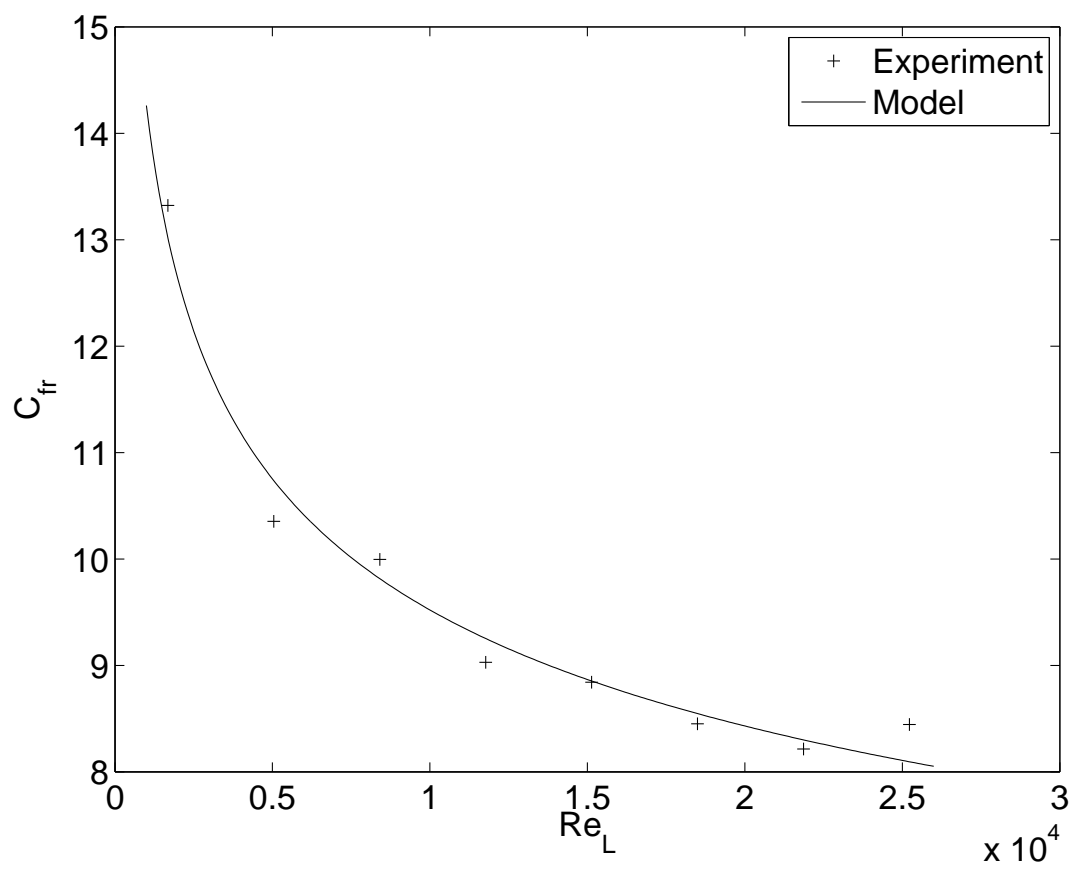

Figure 7: Variation of the friction coefficient with respect to Reynolds number: $\left(r_{d L}=0.25, P r_{f}=0.7, G r_{f}=3.3510^{6}, A=10, D a=7.310^{-5}\right)$ 


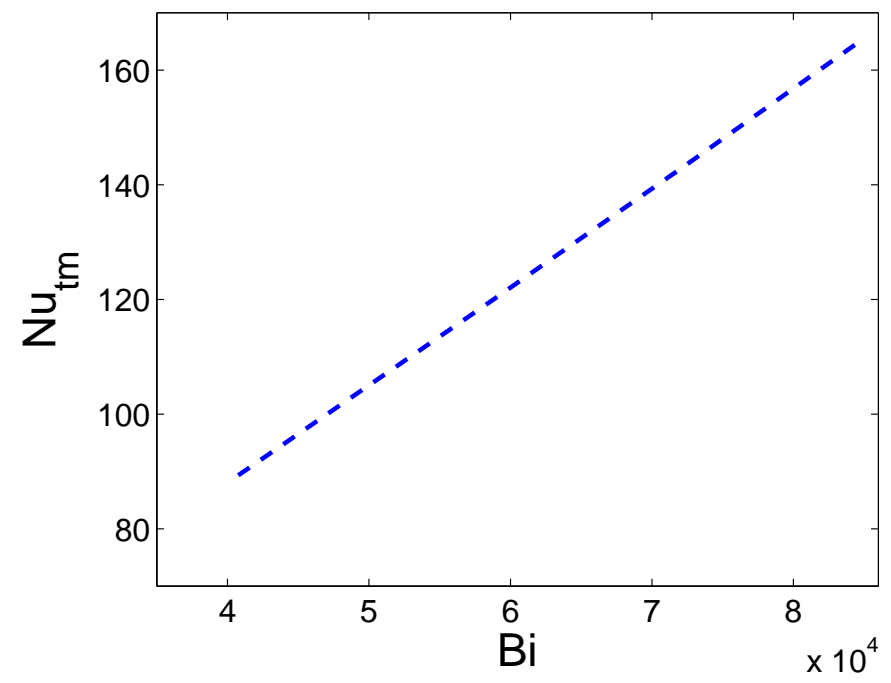

Figure 8: Variation of the mean total Nusselt number with respect to Biot number at constant bead diameter $\left(G r_{f}=10^{7}, P r_{f}=0.7, A=5, \lambda=28.57\right.$, $\left.r_{d L}=0.034, D a=1.310^{-6}, F=0.57\right)$ 


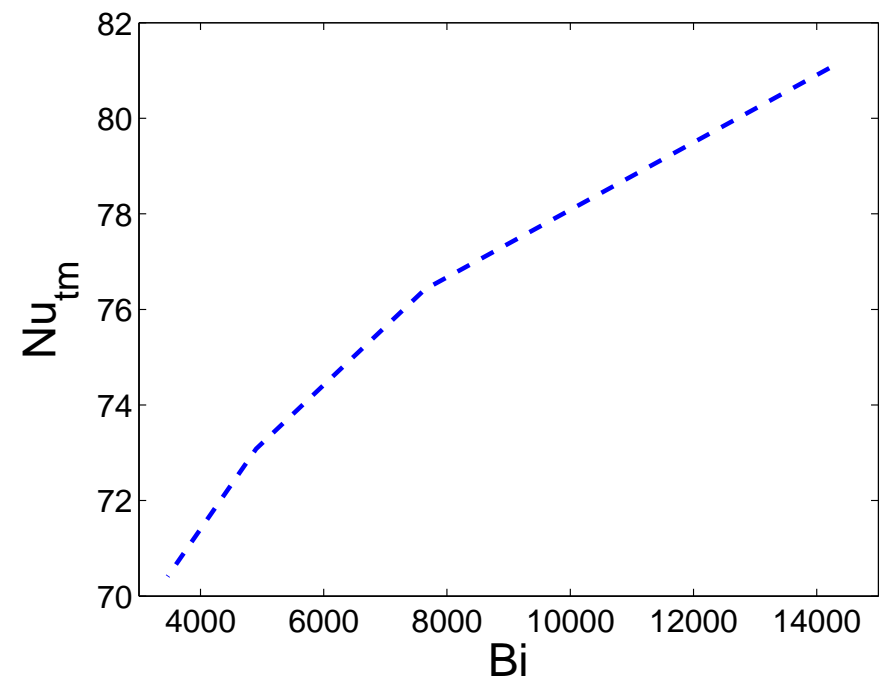

Figure 9: Variation of the mean total Nusselt number with respect to Biot number at constant inlet velocity $\left(G r_{f}=10^{7}, P r_{f}=0.7, A=5, \lambda=28.57\right.$, $\left.\operatorname{Re}_{L}=1500, F=0.57\right)$ 


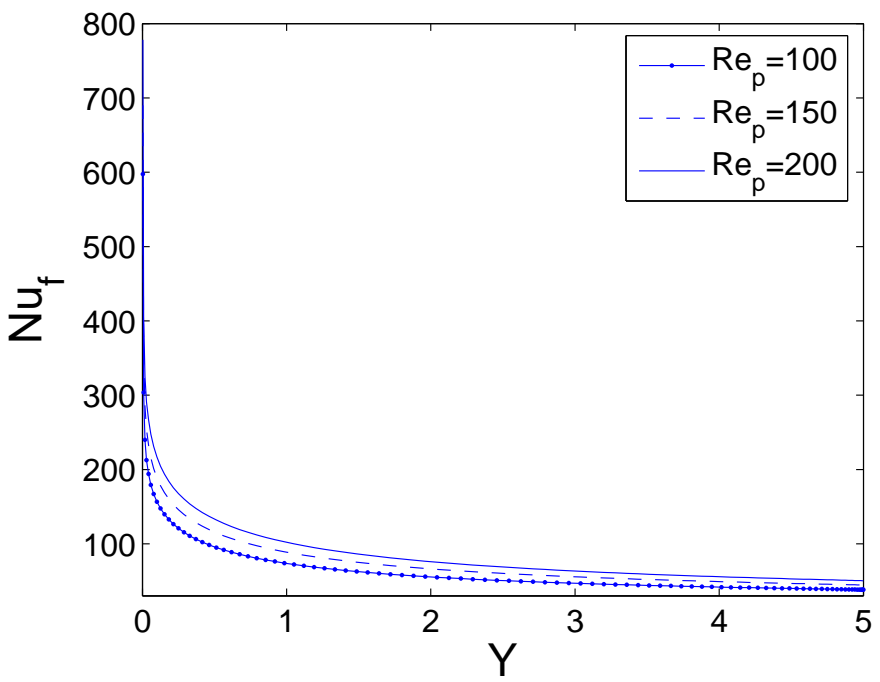

Figure 10: Variation of the local fluid Nusselt number at $X=0$, for different Reynolds numbers $\left(G r_{f}=10^{7}, P r_{f}=0.7, A=5, r_{d L}=0.067, \lambda=28.57\right.$, $\left.D a=5.210^{-6}, F=0.57\right)$ 


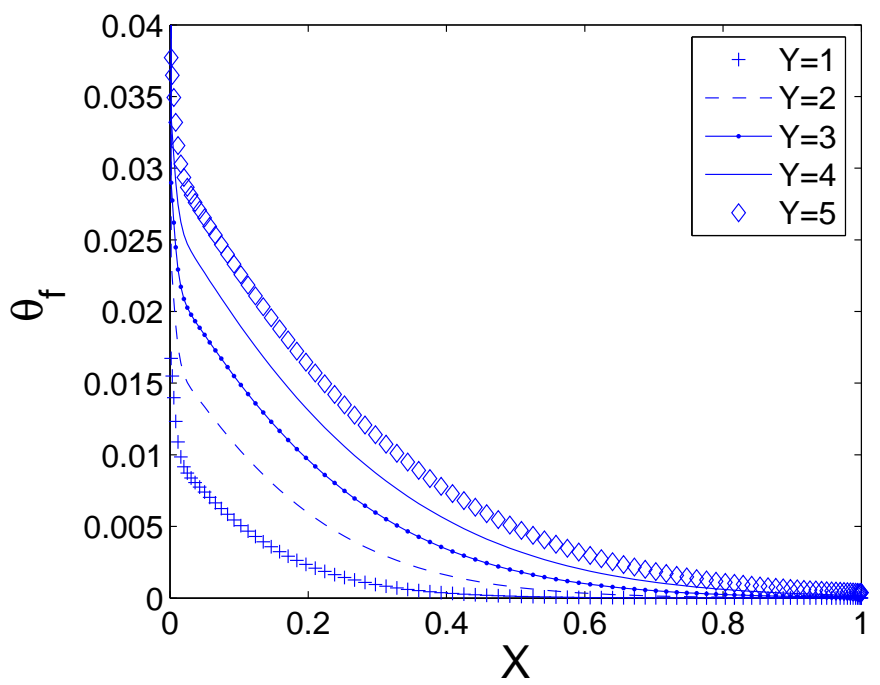

Figure 11: Variation of the temperature of the fluid phase, at different heights in the porous channel $\left(G r_{f}=10^{7}, P r_{f}=0.7, R e_{p}=100, A=5, r_{d L}=0.067\right.$, $\left.D a=5.210^{-6}, F=0.57\right)$ 


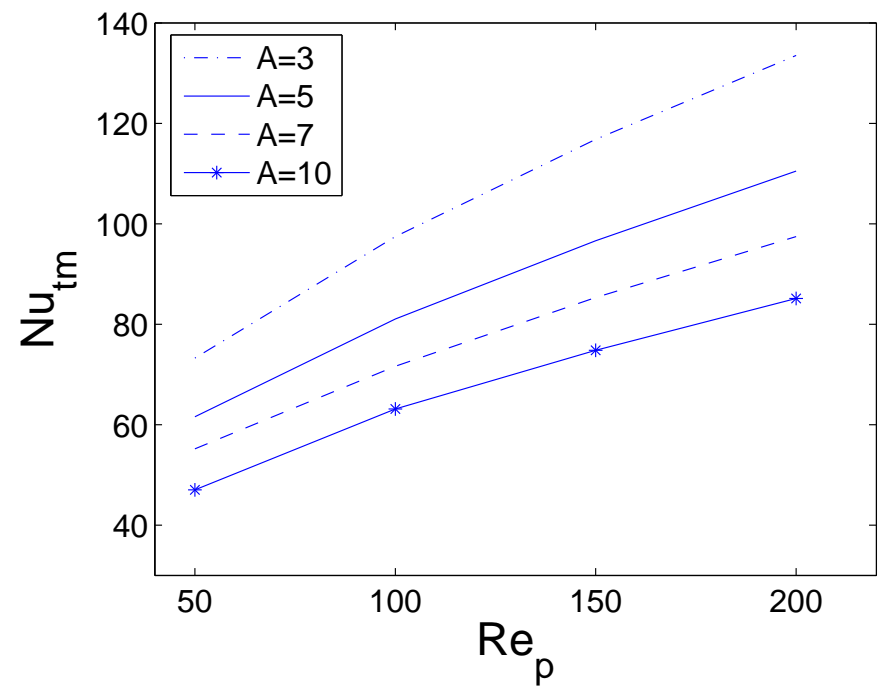

Figure 12: Variation of the mean total Nusselt number with respect to Reynolds number for different form factors $\left(G r_{f}=10^{7}, P r_{f}=0.7, r_{d L}=0.067, \lambda=\right.$ 28.57, $\left.D a=5.210^{-6}, F=0.57\right)$ 


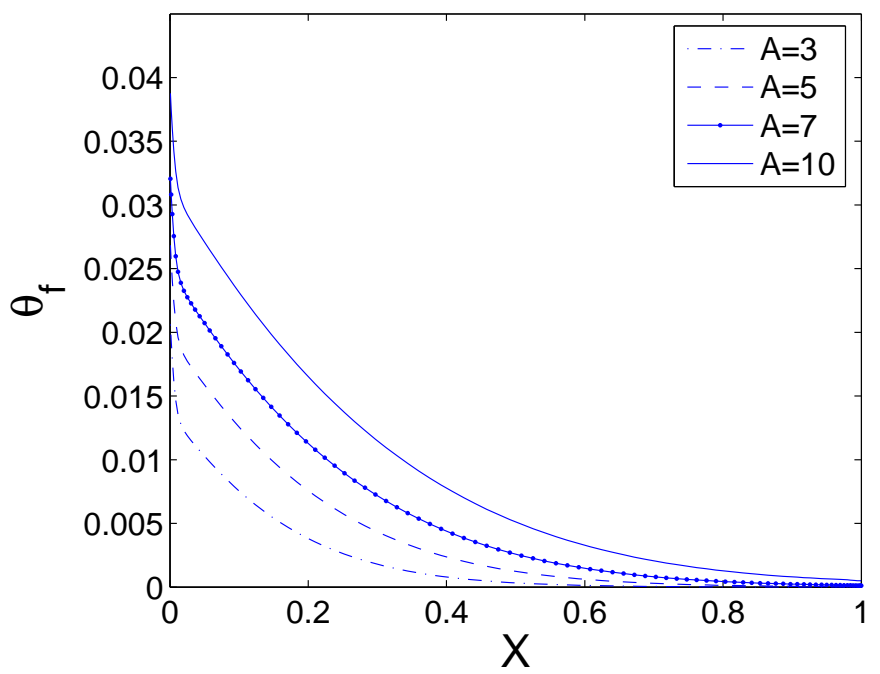

Figure 13: Variation of the temperature of the fluid phase at mid-height, with respect to the width $X$, for different form factors $\left(G r_{f}=10^{7}, P r_{f}=0.7\right.$, $\left.R e_{p}=100, r_{d L}=0.067, D a=5.210^{-6}, F=0.57\right)$ 


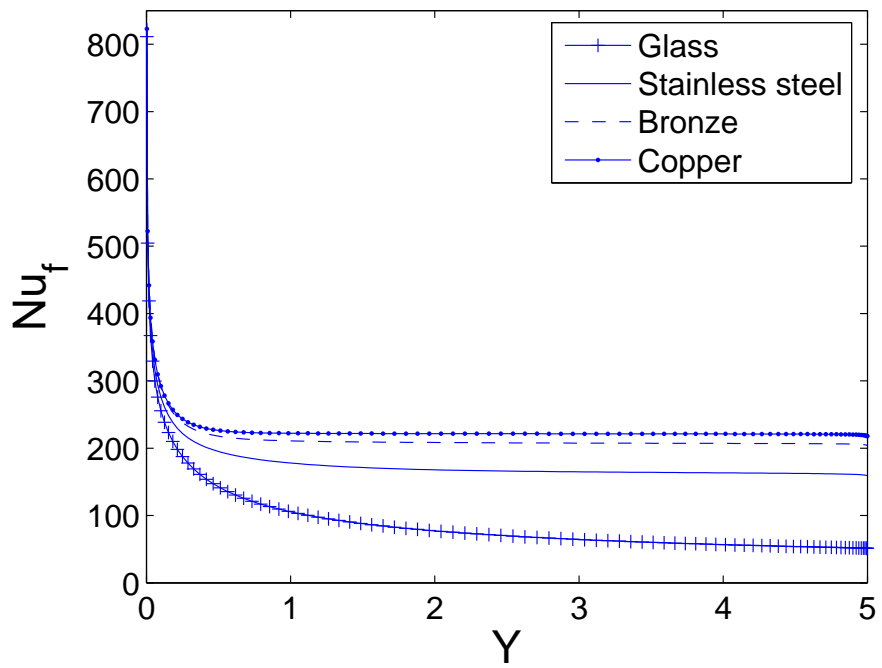

Figure 14: Variation of the local fluid Nusselt number at $X=0$, for different conductivities of beads $\left(G r_{f}=10^{7}, P r_{f}=0.7, A=5, r_{d L}=0.034, R e_{p}=100\right.$, $\left.D a=1.310^{-6}, F=0.57\right)$ 


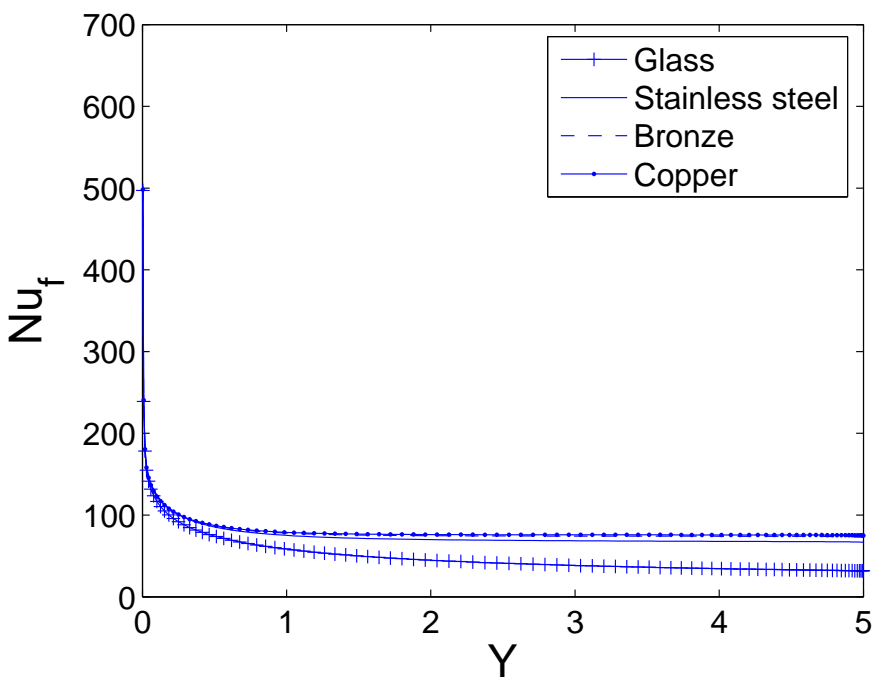

Figure 15: Variation of the local fluid Nusselt number at $X=0$, for different conductivities of beads $\left(G r_{f}=10^{7}, P r_{f}=0.7, A=5, r_{d L}=0.1, R e_{p}=100\right.$, $\left.D a=1.1810^{-5}, F=0.57\right)$ 


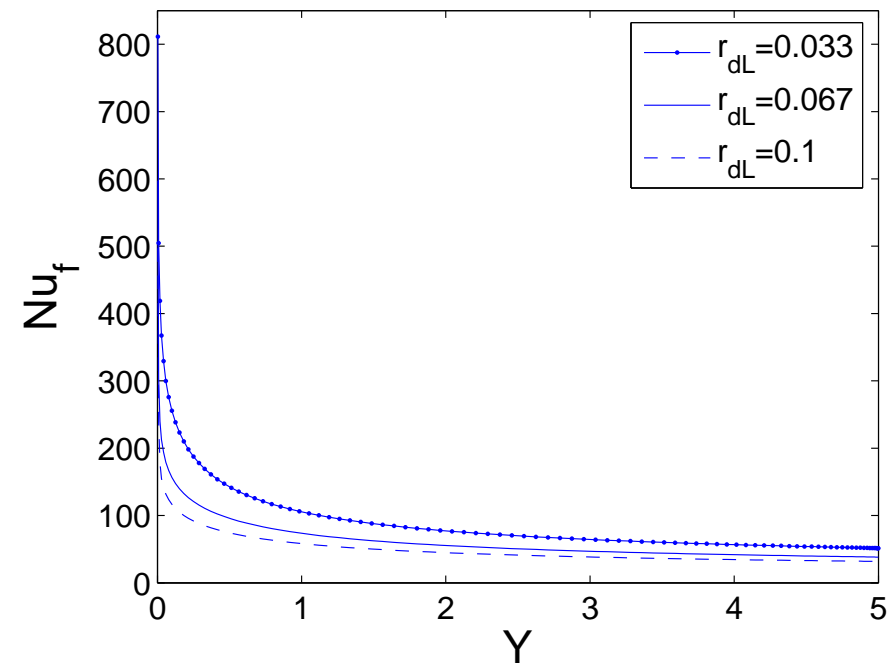

Figure 16: Variation of the local Nusselt number of the fluid at $X=0$, for different diameters of beads $\left(G r=10^{7}, \operatorname{Pr}_{f}=0.7, A=5, R e_{p}=100, \lambda=28.57\right.$, $F=0.57)$ 


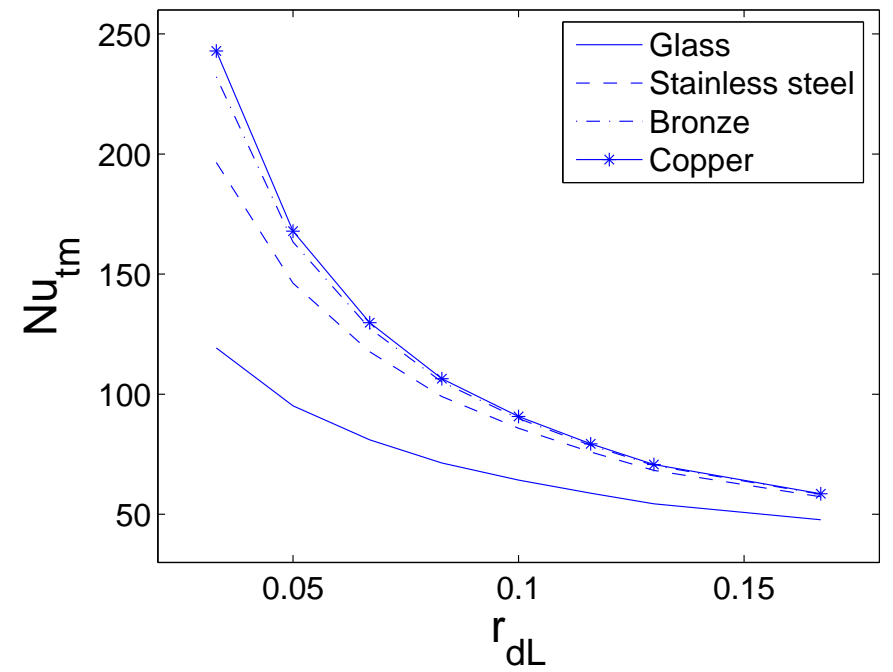

Figure 17: Variation of the mean total Nusselt number for the fluid with respect to the diameter of the beads for different ratios of fluid-solid conductivities $\left(G r_{f}=10^{7}, \operatorname{Pr}_{f}=0.7, R e_{p}=100, A=5, F=0.57\right)$ 


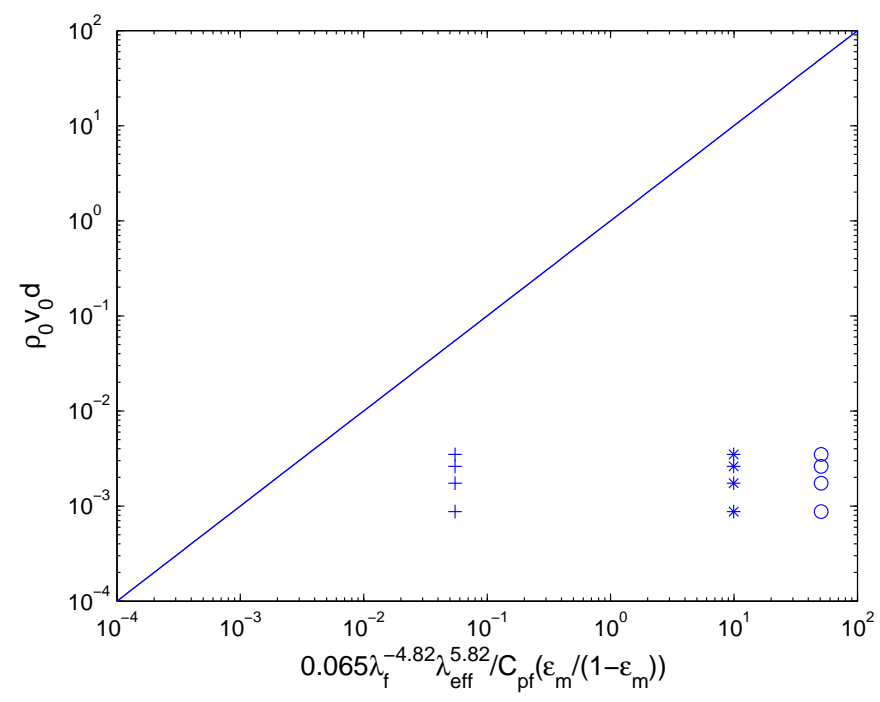

Figure 18: Verification of (Jiang et al., 1999b) criterion for air-glass $(+)$, airstainless steel $(*)$, air-bronze $(\mathrm{o})$ 


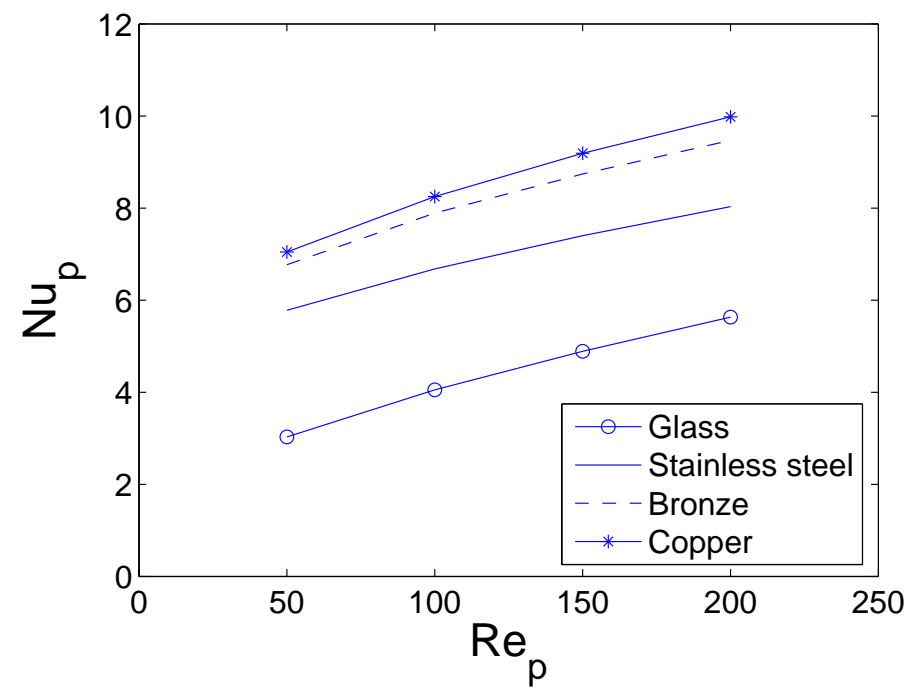

Figure 19: Variation of the mean total Nusselt number with respect to Reynolds number for different conductivities of beads $\left(G r_{f}=10^{7}, P r_{f}=0.7, A=5\right.$, $\left.r_{d L}=0.034, D a=1.310^{-6}, F=0.57\right)$ 


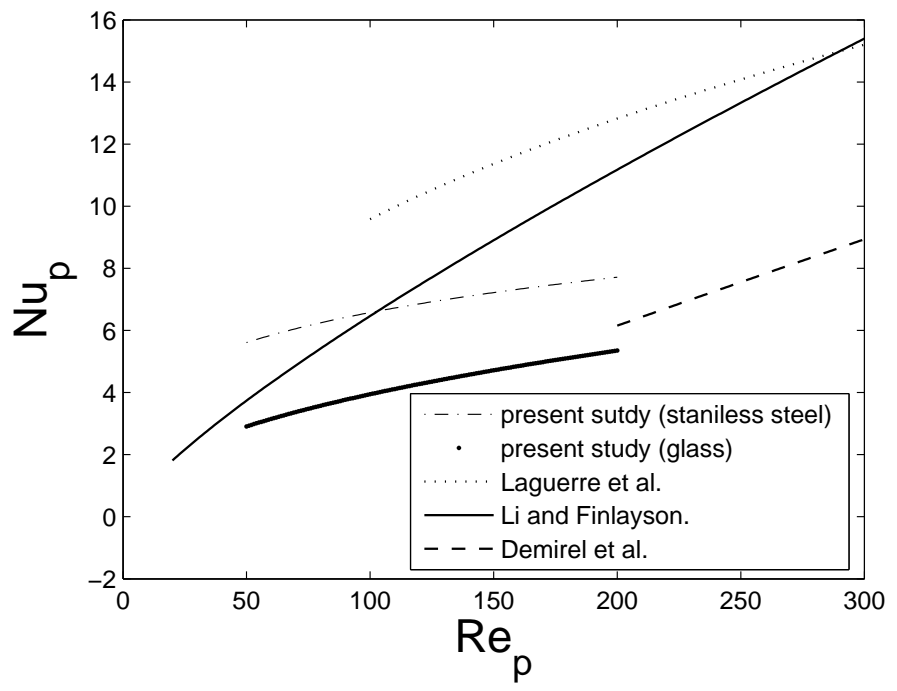

Figure 20: Comparison of correlation equations concerning Nusselt number with respect to Reynolds number 


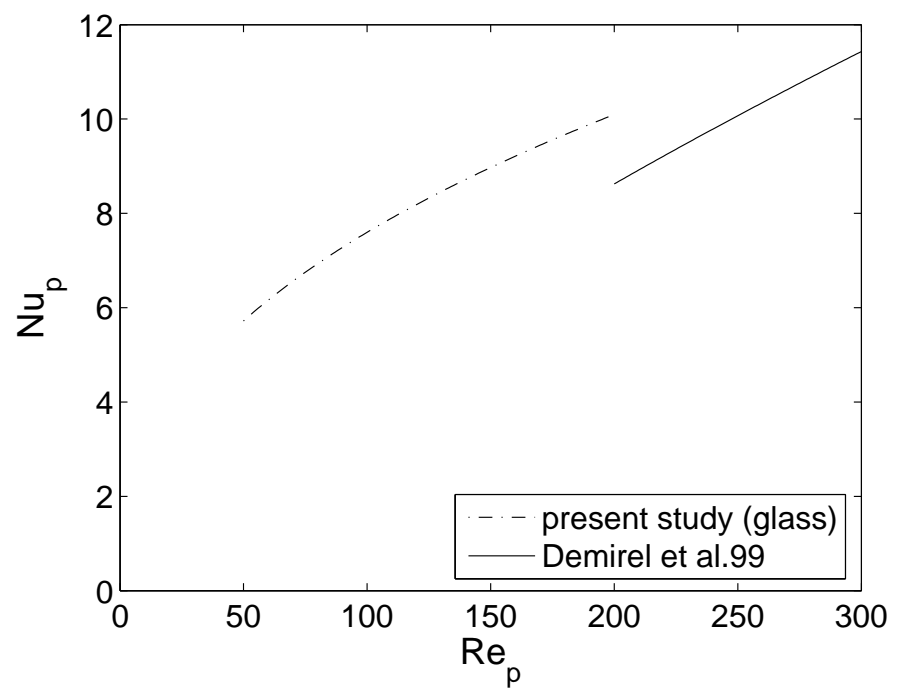

Figure 21: Correlation equations for $N u_{p}$ with respect to $R e_{p}$ and $d / L$ or $d / d_{e}$ 